\title{
Investigation of Equatorial Medium Earth Orbits for Space Solar Power
}

\author{
Michael A. Marshall, Richard G. Madonna, Senior Member, IEEE, and Sergio Pellegrino
}

\begin{abstract}
Most existing space solar power concepts place one or more power stations in geosynchronous Earth orbit (GEO). However, due to the limited availability of GEO orbital slots, it may not be feasible to locate a power station in GEO. To overcome this limitation, this paper presents a system analysis for a space solar power system that incorporates a constellation of power stations in a $20,184 \mathrm{~km}$ altitude equatorial medium Earth orbit (MEO). The orbiting power stations are based on the Caltech Space Solar Power Project architecture. The constellation consists of multiple power stations in a shared equatorial MEO each transmitting to a non-equatorial receiving station. The analysis assumes a one-to-one correspondence between the number of power stations and the number of ground stations. Like a GEO-based system, this constellation architecture enables a MEO-based system to provide near continuous power (outside of eclipse) to each ground station. It is shown that a MEO constellation with three or more power stations provides comparable transmission efficiency to a GEO-based system. The Levelized Cost of Electricity (LCOE) is then computed for MEO systems with three, four, and five power stations and compared to the LCOE for the GEO-based system. Ground station area is identified as a significant contributor to the LCOE for the MEO-based systems. The system analysis shows that a MEO constellation with as few as four power stations has an LCOE comparable to GEO, and hence, it is concluded that MEO is a viable alternative to GEO for space solar power.
\end{abstract}

Index Terms-satellite constellation, geosynchronous Earth orbit, levelized cost of electricity, medium Earth orbit, phased array, photovoltaics, space solar power, space system modeling.

\section{INTRODUCTION}

$\mathbf{S}$ PACE solar power involves collecting sunlight in space and beaming it wirelessly to a terrestrial power grid. Compared to terrestrial solar power, space solar power has two obvious advantages: 1) it decouples power collection from terrestrial weather, diurnal cycles, and seasonal cycles, and 2) it provides the capability to deliver power to virtually any location on Earth at any time, so long as the system is placed in a suitable orbit.

The original idea for space solar power dates back to 1941 [1]. In the years since, researchers have proposed a variety of space solar power concepts. For brevity, we refer readers to the literature for a history of space solar power technologies and concepts [2], [3].

Typically, space solar power involves placing one or more power stations in a geosynchronous Earth orbit (GEO) [4][11], although [5] and [11] also briefly describe low Earth orbit

M. A. Marshall and S. Pellegrino are with the Graduate Aerospace Laboratories, California Institute of Technology, Pasadena, CA, USA e-mails: mmarshall@caltech.edu.sergiop@ caltech.edu

R. G. Madonna is with Systems Engineering Consultants, Columbia, MD, USA e-mail: rmadonna@ caltech.edu

Manuscript recelved XXXXX XX, XXXX; revised XXXXX XX, XXXX.
(LEO) and medium Earth orbit (MEO) concepts. However, overcrowding in GEO [12] makes it problematic to place a large space solar power station there. As a result, there is a distinct need to investigate the feasibility of non-geosynchronous orbits for space solar power. This paper attempts to respond to this need by considering a concept for a constellation of power stations in MEO. For the purpose of this paper, a constellation is defined as a system comprised of two or more power stations.

MEO-based space solar power is potentially advantageous because MEO is largely unoccupied (with several notable exceptions, e.g., GPS) and the launch cost per kilogram of payload is significantly lower for MEO compared to GEO. Additionally, like its GEO counterpart, a properly designed constellation of MEO-based power stations can provide continuous power outside of eclipse to a terrestrial power grid. Despite its advantages, MEO has a critical disadvantage, namely that the radiation environment in MEO is considerably harsher than the radiation environment in GEO [13].

Radiation hardening aside, assessing the viability of MEObased space solar power requires assessing whether a system in MEO is cost effective compared to a baseline system in GEO. This is the purpose of this paper. If the MEO system is cost effective, it provides strong motivation for investigating how best to radiation harden a space solar power satellite for the MEO environment. It likewise provides strong motivation for investigating other unique aspects of a MEO system, like the risks associated with beaming power from a power station moving relative to its ground station. While significant, these studies are outside the scope of this paper.

To assess the relative economic viability of MEO, this paper extends the system model discussed in [14] to address some of the unique aspects of MEO-based constellations of space solar power stations. The system model is applied to a baseline system in GEO and three different constellations in MEO to understand the effects of constellation size on system cost and performance. We consider space solar power satellites based on the concept proposed by the Space Solar Power Project (SSPP) at the California Institute of Technology (Caltech) 1

This paper is organized as follows: Sec. II describes the space solar power concept proposed by the Caltech SSPP. Sec. III investigates power-optimal guidance for a constellation of MEO-based power stations based on the Caltech SSPP architecture. Power-optimal guidance drives transmission performance for the Caltech SSPP concept [15]. These guidance results are an important input to the system model introduced

${ }^{1}$ https://www.spacesolar.caltech.edu 


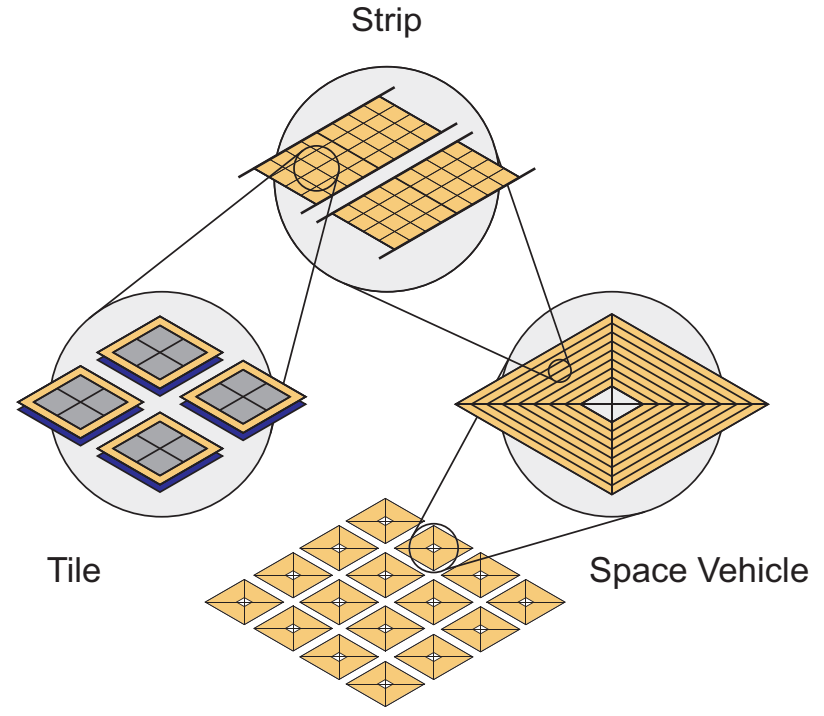

Power Station

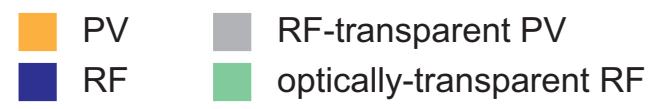

(a)

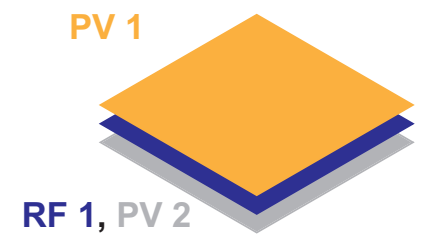

(b)

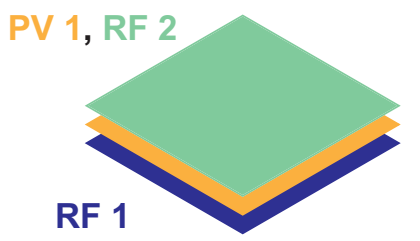

Fig. 1. Modular, scalable power station architecture for the Caltech SSPP concept. The tile is the basic unit of functionality from which the rest of the system scales.

in Sec. IV Sec. V then applies this system model to compare three MEO-based constellations with a baseline power station in GEO. Finally, Sec. VI discusses the paper's conclusions.

\section{Overview of the Caltech Space Solar Power CONCEPT}

We briefly describe the concept being pursued by the Caltech SSPP in order to provide context for what follows. Our concept has matured over the past several years, but the basic architecture is unchanged.

Our approach is predicated on reducing the mass of the space-based power station to achieve an economically viable Levelized Cost of Electricity (LCOE). The LCOE is the average cost of the electricity delivered to the power grid over a system's lifetime. Hence, it provides a metric for comparing the economic viability of power generation systems; for additional details, see Sec. IV] Previous approaches [2], [3] relied on the development of new launch systems capable of reducing launch costs by a factor of 10 or more over current launch costs. By reducing the mass of each power station by as much as a factor of 10 over previous approaches, we can achieve the same end result without relying on the availability of new launch systems. To our knowledge, aside from our previous work [14], the only space solar power concept to utilize LCOE as a performance metric is [11]. However, unlike [11], which simply lists an LCOE estimate with no justification, our LCOE estimates are fully traceable, i.e., we provide a detailed description of our LCOE model and its inputs.

The Caltech SSPP employs the modular, scalable power station architecture (Fig. 11) described in [9]. The tile is the smallest functional unit and contains the photovoltaic (PV) cells and everything else required to convert incident solar power to radio frequency (RF) power and transmit it to a
Fig. 2. Layering schematics for (a) a dual-sided PV, single-sided RF tile, and (b) a single-sided PV, dual-sided RF tile. The tile is a thin $(<2 \mathrm{~mm})$ multi-layer sandwich structure that converts solar power to radiated RF power.

ground station. Tiles are multi-layer, flexible sandwich structures; each layer has one or more functions. The SSPP version of the tile is derived from an earlier Caltech project [16][18], although it is noted that several other research groups have also developed and demonstrated similar concepts at the laboratory scale [19], [20]. Likewise, it is noted that while there have been several terrestrial demonstrations of wireless power transmission technologies [21]-[23], wireless power transmission from an orbiting spacecraft to an earthbound receiver has not yet been demonstrated.

Fig. 2 schematically depicts two tile architectures: a dualsided PV, single-sided RF tile and a single-sided PV, dual-sided RF tile. The dual-sided PV, single-sided RF and single-sided PV, dual-sided RF tiles incorporate RF-transparent PV and optically-transparent RF surfaces, respectively, to enable dualsided operations. As discussed in [15], dual-sided operations increase the overall efficiency of the power station by as much as $50 \%$ by allowing the system to transmit when the Earth is between the Sun and the power station. For an overview of the current state-of-the-art for optically-transparent antennas, see [24] and the references therein. Of note is that opticallytransparent antennas with optical transmission efficiencies of approximately $90 \%$ have been demonstrated [25], although measured values from experimental demonstrations are typically in the range from approximately $30 \%$ to $70 \%$; see e.g., [26]-[28]. The development of practical RF-transparent PV and optically-transparent RF surfaces is the subject of ongoing research at Caltech. The RF layer is responsible for converting direct current (DC) electrical power into RF power, RF phasing, and precision timing. It also contains patch antennas spaced at half-wavelength intervals.

The tiles are integrated into an ultralight, planar carbon fiber structure that consists of longerons and battens supported by deployable booms [9], [30], [31]. The resulting structure - 


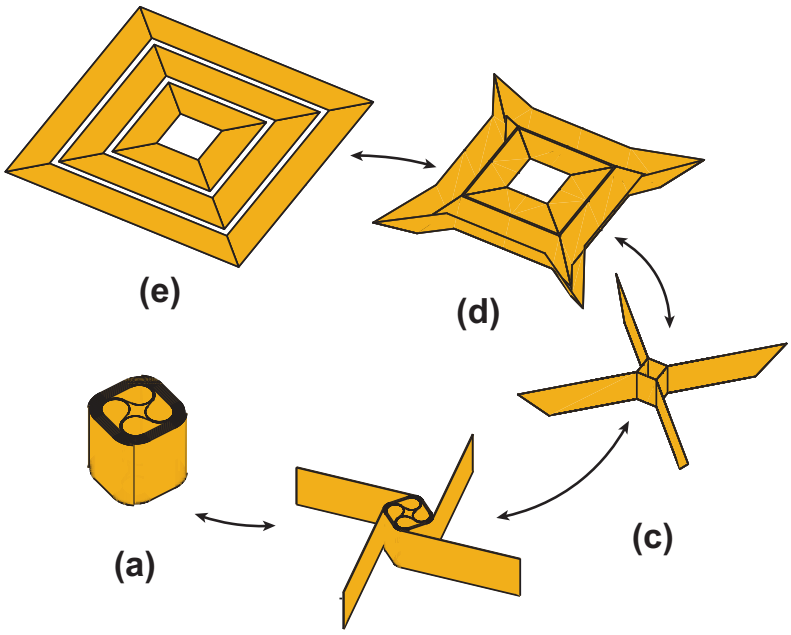

(b)

Fig. 3. Space vehicle packaging concept. The strips are folded and rolled into the stowed configuration. Booms are along the diagonals and longerons run parallel to the strips. Figure adapted from [29].

our payload - is a phased array integrated with a spacecraft bus that provides the required navigation, attitude determination and control, communications, and command and control functions. As in [9], the payload can be z-folded and rolled for stowage prior to launch (Fig. 3] [9], [29], [31], allowing us to package a nominally $60 \mathrm{~m} \times 60 \mathrm{~m}$ payload into a cylindrical volume approximately $2.2 \mathrm{~m}$ in diameter and $1.8 \mathrm{~m}$ in height. These stowed dimensions are based on extrapolating the results of packaging studies on small-scale laboratory models [9], [31]. The payload plus spacecraft bus constitute a space vehicle (also variously referred to as a satellite).

A space vehicle is the basic unit of functionality for the space segment. A power station is a collection of one or more space vehicles arranged to form a very large phased array. The number of space vehicles per power station is determined by the average power delivered to an electrical grid, as discussed in Sec. IV] Phasing not only permits us to focus the RF energy (if the ground station is in the Fresnel zone of the array) or create a diffraction-limited spot on the ground (if the ground station is in the far-field of the array), but it also permits us to point the RF energy to any region on Earth's surface visible to the power station.

To create this large aperture phased array, many space vehicles must maintain precise spatial positions with respect to each other. Our current concept envisions using autonomous maneuvering space vehicles to formation fly in very close proximity [32], [33], eliminating the need for mechanical interconnections between space vehicles. Importantly, to eliminate PV shadowing by adjacent space vehicles, the space vehicles must operate in a planar formation [32], [33]. This is an active area of research by us and others, and for the purpose of this paper, we assume proper relative spatial positioning between space vehicles. In particular, this assumption allows us to consider the attitudes of a power station and its constituent space vehicles interchangeably, i.e., the attitude of a power station and its constituent space vehicles are the same regardless of the number of space vehicles per power station.

The planar architecture of the space vehicles couples the orientations of their PV and RF surfaces with respect to the Sun and ground station, respectively. In other words, changing the orientation of the PV surface with respect to the Sun changes the orientation of the RF surface with respect to the ground station and vice versa. Consequently, the power transmitted by a space vehicle depends on its orientations relative to both the Sun and ground station. In turn, the planar space vehicle architecture requires us to develop attitude guidance that maximizes the energy collected and transmitted to Earth. Attitude guidance for a single space vehicle or power station in a circular, equatorial orbit transmitting to an equatorial ground station is discussed in [15]. The following section extends this attitude guidance to constellations of power stations in a shared circular, equatorial orbit transmitting to ground stations at arbitrary latitudes. Due to their higher efficiency, we only consider dual-sided space solar power satellites in this paper.

\section{Power-Optimal Guidance for a Constellation of Space Solar Power Stations}

This section extends the power-optimal guidance in [15] to a constellation of $N$ equally-spaced space solar power stations in a shared circular, equatorial orbit transmitting to $N$ equallyspaced ground stations. The $N$ space solar power stations and $N$ ground stations are equally-spaced in true anomaly and longitude, respectively. We assume a one-to-one correspondence between the number of power and ground stations, i.e., at any given time, each power station transmits to only one ground station and no two power stations simultaneously transmit to the same ground station. We likewise assume that the orientation of each power station is the same as the orientations of each of its constituent space vehicles, as discussed in Sec. II Hence, the power-optimal guidance developed in this section is independent of the number of space vehicles per power station.

Power-optimal guidance provides the orientations of the power stations that maximize the total power (and hence, energy) transmitted to the ground stations [15]. The total transmitted power depends on the geometry of the power station relative to the Sun and the receiving ground station. Power-optimal guidance is used to estimate the average geometric efficiencies required by the LCOE model in Sec. IV The average geometric efficiency is a figure of merit that encapsulates the transmission performance of a power station.

We restrict our study to constellations in a shared circular, equatorial orbit for several reasons. First, for a constellation in an equatorial orbit, precession of the orbit plane does not change the ground station coverage from day-to-day. This reduces the stationkeeping requirements compared to an equivalent constellation in an inclined orbit. Note that quantifying orbit perturbations and estimating stationkeeping requirements are outside the scope of this paper. An equatorial orbit is also advantageous because it enables a power station to provide coverage to ground stations on both sides of the equator. For example, for the $20,184 \mathrm{~km}$ altitude MEO constellations considered in this paper, an equatorial orbit provides 


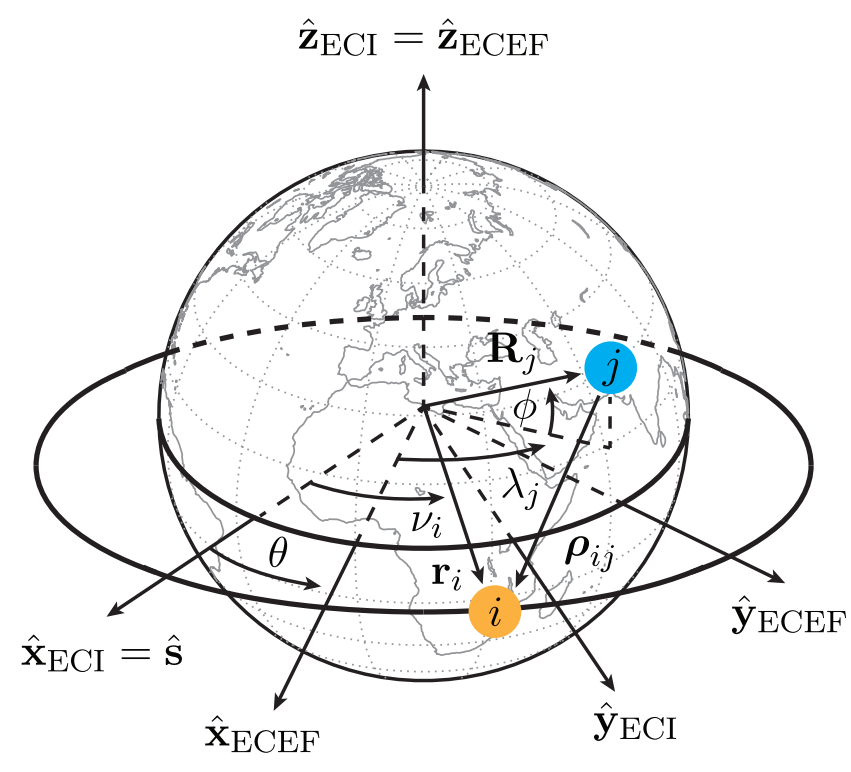

Fig. 4. Reference frame definitions and problem geometry. $i$ and $j$ denote the $i$-th power station and $j$-th ground station, respectively.

coverage between approximately $-40^{\circ}$ and $+40^{\circ}$ latitude. At this altitude, a minimum of three power stations can provide continuous coverage over a ground station, meaning power can be continually transmitted to a ground station provided the overhead power station is not in eclipse. Likewise, a circular orbit simplifies the constellation phasing because each power station has equal coverage over every ground station. In the future, this work can be expanded to consider power stations in inclined orbits and/or constellations with multiple orbit planes, like a Walker constellation [13], but this would require the consideration of higher-order orbital effects.

\section{A. Problem Geometry}

We begin by considering the geometry depicted in Fig. 4 Following [15], we simplify the Earth-Sun geometry by assuming that the Earth's location is fixed at the vernal equinox, meaning we neglect the Sun's apparent motion and assume that the equator is coplanar with a great circle of the Sun. We define an Earth-Centered Inertial (ECI) reference frame with orthonormal basis vectors $\left\{\hat{\mathbf{x}}_{\mathrm{ECI}}, \hat{\mathbf{y}}_{\mathrm{ECI}}, \hat{\mathbf{z}}_{\mathrm{ECI}}\right\}$. The ECI frame is inertially-fixed with its origin at the center of the Earth. At the equinox, the line connecting the center of the Earth with the Sun (the "sun vector," denoted $\hat{\mathbf{s}}$ ) defines $\hat{\mathbf{x}}_{\mathrm{ECI}}$. Earth's rotation axis defines $\hat{\mathbf{z}}_{\mathrm{ECI}}$. The right-hand rule then defines $\hat{\mathbf{y}}_{\mathrm{ECI}}$. $\hat{\mathbf{x}}_{\mathrm{ECI}}$ and $\hat{\mathbf{y}}_{\mathrm{ECI}}$ define Earth's equatorial plane. We use the ECI frame to locate the power stations.

We additionally define a rotating Earth-Centered, EarthFixed (ECEF) reference frame with orthonormal basis vectors $\left\{\hat{\mathbf{x}}_{\mathrm{ECEF}}, \hat{\mathbf{y}}_{\mathrm{ECEF}}, \hat{\mathbf{z}}_{\mathrm{ECEF}}=\hat{\mathbf{z}}_{\mathrm{ECI}}\right\} . \hat{\mathbf{x}}_{\mathrm{ECEF}}=\hat{\mathbf{x}}_{\mathrm{ECI}}$ at time $t=0$. $\hat{\mathbf{x}}_{\mathrm{ECEF}}$ defines the Prime Meridian. The ECEF frame rotates about the $\hat{\mathbf{z}}_{\mathrm{ECI}}$ axis at a rate equal to the rotation rate of Earth about its axis, $\omega_{\oplus}$, and has its origin at the center of the Earth. The transformation from the ECEF frame to the ECI frame is

$$
{ }^{\mathrm{ECI}} \mathcal{R}^{\mathrm{ECEF}}=\left[\begin{array}{ccc}
\cos (\theta) & -\sin (\theta) & 0 \\
\sin (\theta) & \cos (\theta) & 0 \\
0 & 0 & 1
\end{array}\right]
$$

where $\theta$ is the Earth rotation angle (ERA) given by

$$
\theta(t)=\omega_{\oplus} t .
$$

We use the ECEF frame to locate the ground stations. This framework allows us to make relative comparisons between different space solar power systems.

The $N$ power stations are in a shared circular, equatorial MEO with altitude $h$. The $i$-th power station's position is

$$
\mathbf{r}_{i}^{\mathrm{ECI}}=r\left[\begin{array}{c}
\cos \left(\nu_{i}\right) \\
\sin \left(\nu_{i}\right) \\
0
\end{array}\right], \quad i=1,2, \ldots, N
$$

where $r=R_{\oplus}+h, R_{\oplus}$ is Earth's mean equatorial radius, $\nu_{i}$ is the $i$-th power station's true anomaly given by

$$
\nu_{i}(t)=\nu_{i}(0)+n t,
$$

and $\nu_{i}(0)$ is the $i$-th power station's true anomaly at time $t=0$. $n=\sqrt{\mu / r^{3}}$ is the orbit's mean motion where $\mu$ is Earth's standard gravitational parameter. We assume that $\nu_{1}(0)=0$.

We additionally assume that the $N$ ground stations are equally-spaced longitudinally and at the same latitude. The position of the $j$-th ground station in the ECEF frame is

$$
\mathbf{R}_{j}^{\mathrm{ECEF}}=R_{\oplus}\left[\begin{array}{c}
\cos \left(\lambda_{j}\right) \cos (\phi) \\
\sin \left(\lambda_{j}\right) \cos (\phi) \\
\sin (\phi)
\end{array}\right], \quad j=1,2, \ldots, N
$$

where $\phi$ and $\lambda_{j}$ are its latitude and longitude, respectively. Transforming (5) from the ECEF frame to the ECI frame with (1) yields

$$
\mathbf{R}_{j}^{\mathrm{ECI}}=R_{\oplus}\left[\begin{array}{c}
\cos \left(\theta+\lambda_{j}\right) \cos (\phi) \\
\sin \left(\theta+\lambda_{j}\right) \cos (\phi) \\
\sin (\phi)
\end{array}\right]
$$

We define the slant vector, pointing from the $j$-th ground station to the $i$-th power station:

$$
\boldsymbol{\rho}_{i j}=\mathbf{r}_{i}-\mathbf{R}_{j}
$$

with magnitude

$$
\rho_{i j}=\sqrt{r^{2}+R_{\oplus}^{2}-2 r R_{\oplus} \cos \left(\Omega_{i j}\right)}
$$

where $\Omega_{i j}$ is the compound angle defined by

$$
\cos \left(\Omega_{i j}\right)=\frac{\mathbf{r}_{i} \cdot \mathbf{R}_{j}}{r R_{\oplus}}=\cos (\phi) \cos \left(\theta+\lambda_{j}-\nu_{i}\right) .
$$

Next, we evaluate the dot product $\mathbf{R}_{j} \cdot \boldsymbol{\rho}_{i j}$ to determine the elevation angle of the $i$-th power station relative to the $j$-th ground station. Using the identity $\cos \left(\pi / 2-\delta_{i j}\right)=\sin \left(\delta_{i j}\right)$, it follows that

$$
\sin \left(\delta_{i j}\right)=\frac{-R_{\oplus} / r+\cos \left(\Omega_{i j}\right)}{\sqrt{1+\left(R_{\oplus} / r\right)^{2}-2\left(R_{\oplus} / r\right) \cos \left(\Omega_{i j}\right)}} .
$$

Solutions to 10 for $\delta_{i j}$ are non-unique because $\delta_{i j}$ 's quadrant is undefined. However, in the usual azimuth-elevation coordinate system [34], $\delta_{i j}$ is restricted to the interval $\left[0^{\circ}, 90^{\circ}\right]$ and the azimuth angle is defined in the interval $\left[0^{\circ}, 360^{\circ}\right)$. Together, these azimuth and elevation angle definitions provide full hemispherical access above a given point on Earth's 


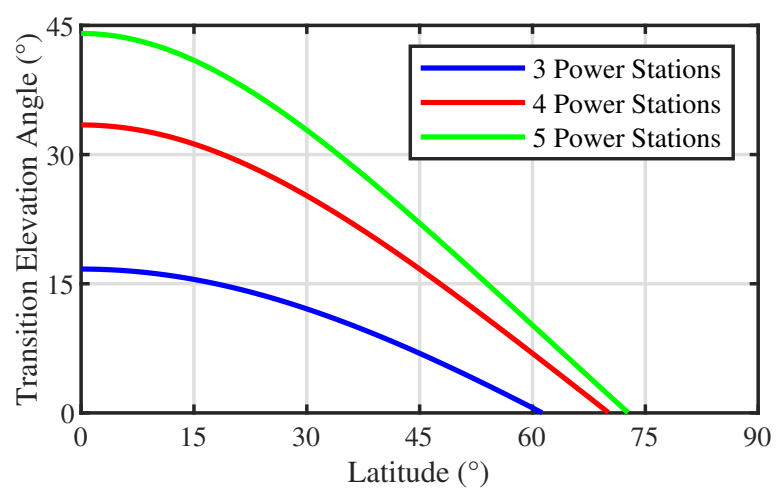

Fig. 5. Transition elevation angle $\delta_{t}$ as a function of latitude and constellation size for a $20,184 \mathrm{~km}$ altitude circular equatorial orbit.

surface. Thus, we can invert (10) to find a unique solution for $\delta_{i j}$ by restricting $\delta_{i j}$ to the first quadrant.

We use (10) to determine the elevation angle when the $i$-th power station stops transmitting to the $j$-th ground station and begins transmitting to the $(j+1)$-th ground station. We refer to this as the transition elevation angle, $\delta_{t}$. For $N$ equally-spaced power stations, the angular spacing between adjacent stations is $2 \pi / N$. As a result, we compute $\delta_{t}$ by evaluating 10 with $\Omega_{i j}=\Omega_{t}$ where

$$
\cos \left(\Omega_{t}\right)=\cos (\phi) \cos (\pi / N) .
$$

Fig. 5 plots $\delta_{t}$ as a function of latitude and constellation size for the 20,184 km altitude circular equatorial orbit considered in this case study. From Fig. 5, we see that $\delta_{t}$ decreases as latitude increases and that increasing the constellation size increases $\delta_{t}$. The dependencies of $\delta_{t}$ on latitude and constellation size have important implications for ground station sizing because the size of the projection of the transmitted RF beam on the Earth's surface increases as elevation angle decreases.

A power station can only transmit to a ground station for elevation angles that exceed some angle $\delta_{h}>0$ above the horizon. Thus, the minimum allowable elevation angle is given by

$$
\delta_{\min }=\max \left\{\delta_{t}, \delta_{h}\right\} .
$$

Since we assume $\delta_{h}>0$, a power station never transmits horizon-to-horizon.

There are two conditions that must be satisfied for a power station to be able to transmit to a ground station: 1) the elevation angle exceeds the minimum elevation angle, i.e., $\delta_{i j}>\delta_{\min }$ for some $i, j=1,2, \ldots, N$, and 2) the power station is not in eclipse. Following [15], we model eclipse as a binary on/off using a conical projection method that ignores penumbral shadowing [35]. For a 20,184 km altitude circular equatorial MEO at the vernal equinox, this results in an eclipse period of approximately 54 minutes per 12hour orbit per power station. Both the equatorial MEO and GEO are predominantly sunlit orbits with eclipse periods restricted to the vicinities of the equinoxes. Consequently, the vernal equinox represents the worst-case eclipse conditions for both MEO and GEO and provides conservative, lower bound estimates for the resulting geometric efficiencies.

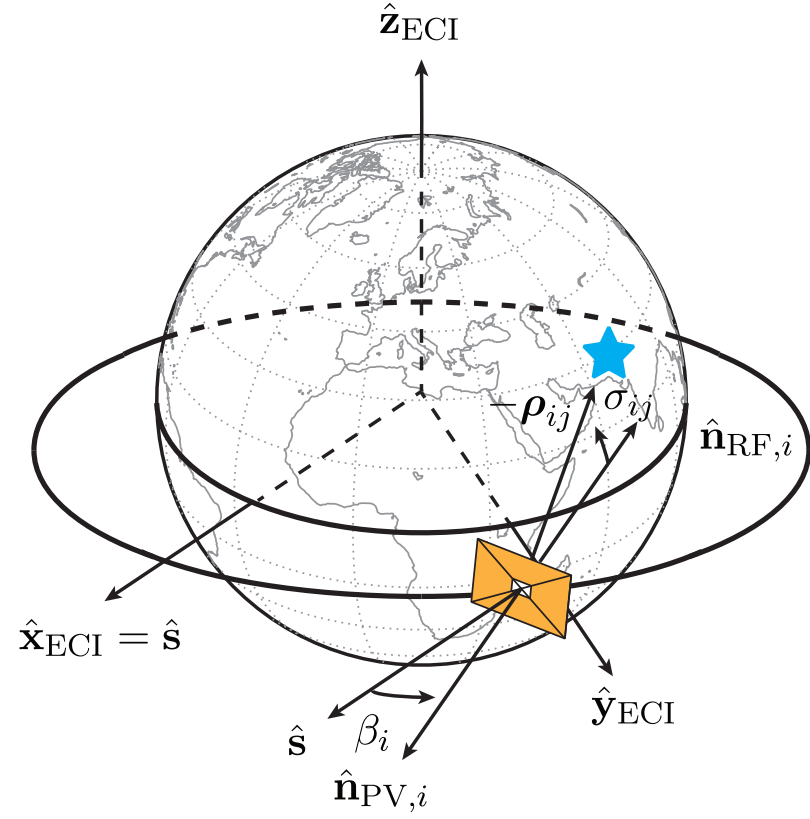

Fig. 6. Sun and squint angle definitions. $\hat{\mathbf{n}}_{\mathrm{PV}, i}$ and $\hat{\mathbf{n}}_{\mathrm{RF}, i}=-\hat{\mathbf{n}}_{\mathrm{PV}, i}$ denote the outward normal vectors from the $i$-th power station's primary PV and RF surfaces.

The transmitted power evaluated in Sec. III-B depends on the problem geometry (Fig. 4) through the sun angle $\beta$ and squint angle $\sigma$ defined in Fig. 6. The sun and squint angles describe the power station's orientation with respect to the Sun and receiving ground station, respectively. Specifically, $\beta$ is the angle between $\hat{\mathbf{n}}_{\mathrm{PV}}$ and the Sun, and $\sigma$ is the angle between $\hat{\mathbf{n}}_{\mathrm{RF}}=-\hat{\mathbf{n}}_{\mathrm{PV}}$ and the ground station. $\hat{\mathbf{n}}_{\mathrm{PV}}$ and $\hat{\mathbf{n}}_{\mathrm{RF}}$ are the outward normal vectors from the power station's primary PV and RF surfaces. To first-order, the efficiencies of the power station's PV and RF surfaces are only functions of $\beta$ and $\sigma$, i.e., they have no radial (azimuthal) dependencies. Hence, we can use either of the two outward normal vectors to describe the power station's attitude, and this attitude can be parameterized as a function of either $\beta$ or $\sigma$.

We use the geometry in Fig. 6 to derive a relationship between $\beta$ and $\sigma$ which is used to constrain the power maximization problem in Sec. III-C. By assuming that the power station is constrained to only rotate about $\hat{\mathbf{z}}_{\mathrm{ECI}}$, we can express the outward normal from the $i$-th power station's primary $\mathrm{PV}$ surface in terms of $\beta_{i}$ as

$$
\hat{\mathbf{n}}_{\mathrm{PV}, i}^{\mathrm{ECI}}=\left[\begin{array}{c}
\cos \left(\beta_{i}\right) \\
\sin \left(\beta_{i}\right) \\
0
\end{array}\right] .
$$

Using Fig. 6, it follows that

$$
\cos \left(\sigma_{i j}\right)=\frac{\hat{\mathbf{n}}_{\mathrm{PV}, i} \cdot \boldsymbol{\rho}_{i j}}{\rho_{i j}} .
$$

which relates the sun and squint angles through the slant vector. Since the slant vector depends on the power station's orbital position, (14) relates the power station's orbit and attitude. For a given sun angle, we can use (14) to compute the corresponding squint angle or vice versa. Note that (14) can be 

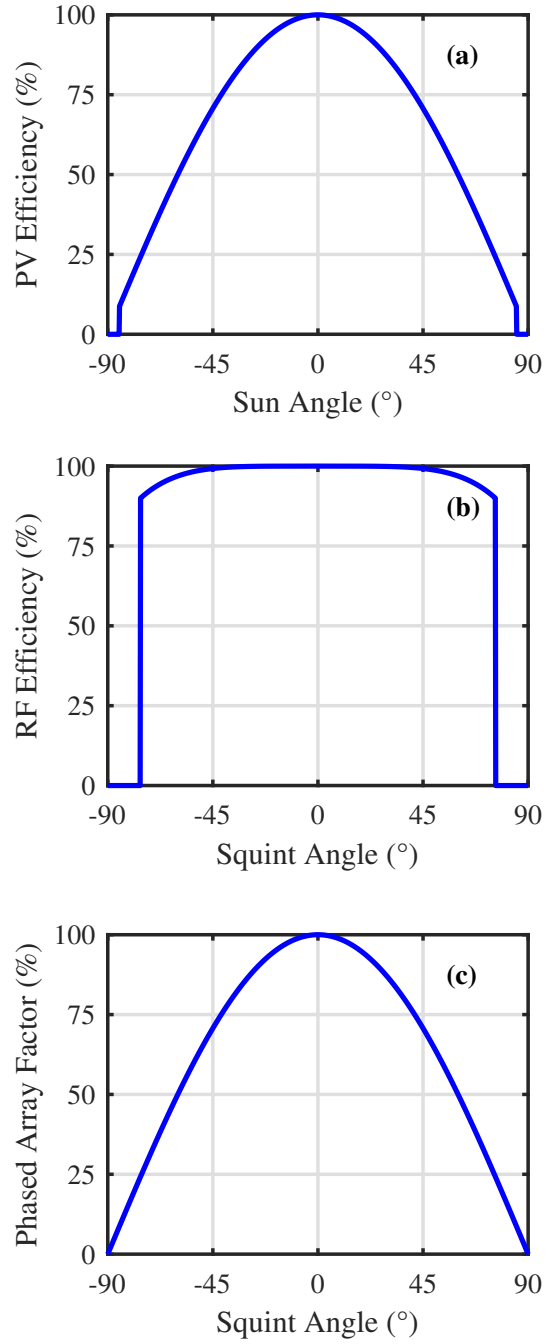

Fig. 7. (a) PV efficiency function for planar PV cells, (b) RF efficiency function for near-isotropic patch antennas, and (c) phased array factor.

extended to non-equatorial orbits by appropriately re-defining $\hat{\mathbf{n}}_{\mathrm{PV}, i}$ in $(13)$.

\section{B. Transmitted Power and Geometric Efficiency}

Following [15], we evaluate the total power transmitted by a single power station in terms of the sun angle $\beta$ and squint angle $\sigma$ using the expression:

$$
P_{t}(\beta, \sigma)=\eta_{\mathrm{PV}} \eta_{\mathrm{DC}-\mathrm{RF}} \eta_{\mathrm{Tx}} A_{\mathrm{PV}} \operatorname{AM} 0 \mathrm{PV}(\beta) \operatorname{RF}(\sigma) \operatorname{AF}(\sigma)
$$

where $P_{t}=0$ if either $\delta<\delta_{\min }$ or if the power station is in eclipse and:

$\eta_{\mathrm{PV}} \quad$ is the optical-to-electrical power conversion efficiency of the PV cells,

$\eta_{\mathrm{DC}-\mathrm{RF}}$ is the DC-to-RF conversion efficiency of the system,

$\eta_{\mathrm{Tx}} \quad$ is the antenna transmit efficiency due to impedance mismatching between the RF source and antenna,

$A_{\mathrm{PV}} \quad$ is the total effective area of the PV cells,

AM0 is $1,366 \mathrm{~W} / \mathrm{m}^{2}$ solar insolation (also commonly referred to as solar irradiance),

$\mathrm{PV}(\beta)$ is the efficiency of the PV cells as a function of sun angle,
$\mathrm{RF}(\sigma)$ is the efficiency of a single antenna in the phased array as a function of squint angle, and

$\operatorname{AF}(\sigma)$ is the phased array factor used to account for the interactions between antennas in the phased array [36].

In general, $\eta_{\mathrm{PV}}, \eta_{\mathrm{DC}-\mathrm{RF}}$, and $\eta_{\mathrm{Tx}}$ are complicated functions of voltage, current, temperature, etc. However, to first-order, they can be approximated as constants, as is done here. Additionally, we use the $\mathrm{PV}$ efficiency $\mathrm{PV}(\beta)$, antenna efficiency $\mathrm{RF}(\sigma)$, and phased array factor $\operatorname{AF}(\sigma)$ from [15], reproduced in Figs. 7a, 7b, and 7c, respectively.

We subsequently define a normalized transmitted power that only depends on the problem geometry, as follows:

$G(\beta, \sigma)=\frac{P_{t}(\beta, \sigma)}{\eta_{\mathrm{PV}} \eta_{\mathrm{DC}-\mathrm{RF}} \eta_{\mathrm{Tx}} A_{\mathrm{PV}} \mathrm{AM} 0}=\operatorname{PV}(\beta) \operatorname{RF}(\sigma) \operatorname{AF}(\sigma)$.

$G$ is referred to as the geometric efficiency because it captures all the geometry-dependent terms in the transmitted power. Since $G$ is simply a scaling of $P_{t}$, maximizing $G$ maximizes $P_{t}$ and vice versa. The average geometric efficiency for the $i$-th power station transmitting to the $j$-th ground station is then given by

$$
\bar{G}_{i j}=\frac{1}{t_{f}} \int_{0}^{t_{f}} G_{i j}\left(\beta_{i}(t), \sigma_{i j}(t)\right) \mathrm{d} t .
$$

where the duration $t_{f}$ is usually a multiple of the orbit period.

\section{Power-Optimal Guidance Problem for a Constellation}

The power-optimal guidance problem determines the attitude trajectory that maximizes the total power transmitted from a single power station to a single ground station. It is defined as follows [15]:

Problem 1 (Power-Optimal Guidance):

$$
\underset{\beta(t), \sigma(t)}{\operatorname{maximize}} \bar{G}(\beta(t), \sigma(t))
$$

subject to (14). In this section, we adapt Problem 1 to account for the constellations considered in this paper. Specifically, Problem 1 is modified to maximize the average geometric efficiencies (17) for all $N$ power stations in the constellation transmitting to each of the $N$ receiving stations.

We remark that Problem 1 intentionally neglects both orbital maneuvering costs (for formation maintenance and stationkeeping) and attitude maneuvering costs (for slewing and rejecting disturbance torques/desaturating momentum devices). Preliminary studies for space vehicles with modern electric propulsion thrusters for orbit and attitude control have shown that the propellant masses required for orbital [33] and attitude [37] maneuvering in the absence of external perturbations are likely a negligible fraction of the total space vehicle mass over a 10-15 year lifetime. In contrast, the power transmission efficiency (by way of the geometric efficiency) is a significant contributor to the system's LCOE. Consequently, it is reasonable to only consider the geometric efficiency in the guidance formulation at this stage of the design process. Note that quantifying orbit and attitude perturbations, along with any perturbations due to structural flexibility, and accounting for how these perturbations impact the required propellant mass are active areas of research for the Caltech SSPP. 
Compared to [15], a critical difference in the formulation of the power-optimal guidance problem for a constellation is the need for an assignment criterion that determines which power station transmits to which ground station at each instance in time. Our chosen assignment criterion maximizes the elevation angles of each power station relative to each ground station. The specific steps in the assignment process are as follows:

1) Propagate the power and ground station positions over the period of interest (in this case, a sidereal day).

2) Compute the elevation angle profiles of each power station relative to each ground station using (10).

3) Use the criterion $\delta_{i j}>\delta_{\min }$ for $i, j=1,2, \ldots, N$ to uniquely assign each power station to a ground station at each instance in time.

We assume that each power station can instantaneously reorient itself to optimally point towards the next ground station when $\delta_{i j}=\delta_{\min }$.

Once the power station assignments are made, we can define the power-optimal guidance problem for a constellation as follows:

Problem 2 (Power-Optimal Guidance for a Constellation):

$$
\underset{\beta_{i}(t), \sigma_{i j}(t)}{\operatorname{maximize}} \sum_{i=1}^{N} \bar{G}_{i j}\left(\beta_{i}(t), \sigma_{i j}(t)\right), \quad j=1,2, \ldots, N
$$

subject to 14 where $i$ denotes the $i$-th power station and $j$ denotes the $j$-th ground station.

The objective function (19) represents the average geometric efficiency of all $N$ power stations transmitting to the $j$-th ground station and is an input to the LCOE model in Sec. [IV] Solving Problem 2 results in the optimal attitude trajectories and corresponding optimal geometric efficiencies for each power station.

For equally-spaced power and ground stations, our assignment criterion guarantees equal coverage (in time) for each power station over each ground station. Because each ground station sees the same relative geometry between an assigned power station, the Sun, and the ground station at different instances in time, the day-averaged geometric efficiencies are equivalent for all the ground stations. In other words, the instantaneous power delivered to each ground station varies at each instance in time, but the average power transmitted to each ground station over a day is the same among all $N$ ground stations. Therefore, to determine the optimal geometric efficiencies, we can consider only the first power station and the first ground station, and hence, can solve the following simplified optimization problem:

Problem 3 (Optimal Geometric Efficiency for a Constellation):

$$
\underset{\beta_{1}(t), \sigma_{11}(t)}{\operatorname{maximize}} \bar{G}_{11}\left(\beta_{1}(t), \sigma_{11}(t)\right)
$$

subject to (14).

The average geometric efficiency of all $N$ power stations transmitting to any of the $N$ ground stations used as an input to the LCOE model is then given by

$$
\bar{G}^{*}=N \bar{G}_{11}^{*}
$$

where $\bar{G}_{11}^{*}$ is the maximum value of $\bar{G}_{11}$ determined from Problem 3. Henceforth, any references to either the geometric efficiency or the average geometric efficiency are to be understood as referring to the quantity $\bar{G}^{*}$ defined by 21 .

Note that for the purpose of system modeling, we are only interested in the optimal geometric efficiencies, not the corresponding attitude trajectories. As a result, the poweroptimality theorem from [15] tells us that we can neglect the constraints on the sun and squint angles when solving either Problems 2 or 3 for a dual-sided power station.

\section{Average Geometric Efficiencies for a Constellation}

We have solved Problem 3 for a constellation in a $20,184 \mathrm{~km}$ altitude circular equatorial orbit with $N=3,4,5$ to study the sensitivity of the day-averaged geometric efficiency $\bar{G}_{d}^{*}$ to ground station latitude. The day-averaged geometric efficiency $\bar{G}_{d}^{*}$ refers to the quantity $\bar{G}^{*}(21)$ where the timeaverage (17) is evaluated over a sidereal day. A $20,184 \mathrm{~km}$ altitude circular orbit is a 2:1 repeating orbit, i.e., it completes two orbits per sidereal day. As a first-order approximation, we can assume that the system performance does not change dayto-day. Hence, $\bar{G}_{d}^{*}$ provides a useful metric for extrapolating system performance over long durations of time. Whereas in absolute terms this assumption is not correct, it enables a relative comparison between different space solar power systems because the value of $\bar{G}_{d}^{*}$ for each system utilizes the same underlying assumptions.

Fig. 8 depicts the sensitivity of $\bar{G}_{d}^{*}$ to the ground station latitude for constellations of dual-sided power stations in a $20,184 \mathrm{~km}$ altitude circular equatorial orbit with $N=3,4,5$. For comparison, the dashed lines in Fig. 8 denote the value of $\bar{G}_{d}^{*}$ for a single power station in GEO.

There are several important observations from Fig. 8 For one, Fig. 8 a demonstrates that $\bar{G}_{d}^{*}$ decreases slowly as the latitude increases so long as $\delta_{i j}>\delta_{h}$ throughout each ground station pass. The small decrease in $\bar{G}_{d}^{*}$ is due to an increase in squint angle as the latitude increases. If $\delta_{i j}>\delta_{h}$ throughout each ground station pass, the constellation maintains $100 \%$ coverage over all $N$ ground stations outside of eclipse. However, as the ground station latitude increases beyond some critical threshold (determined by the latitude where $\delta_{h}=\delta_{t}$, see Fig. 5p, $\delta_{i j}<\delta_{h}$ for part of each pass. As a result, dead zones appear in each pass where the power station is too low on the horizon to transmit to the ground station. Hence, beyond this critical latitude threshold, the ground station pass duration decreases, resulting in a corresponding decrease in $\bar{G}_{d}^{*}$. Based on Fig. 5. when $\delta_{h}=45^{\circ}, \delta_{h}>\delta_{t}$, irrespective of latitude, which further limits the usable ground station pass duration. This explains the substantial decrease in $\bar{G}_{d}^{*}$ exhibited by Fig. $8 \mathrm{~b}$ relative to Fig. $8 \mathrm{a}$ Additionally, this explains why larger constellations tend to exhibit higher values of $\bar{G}_{d}^{*}$.

Note that the maximum local squint angles on the active RF surfaces for each power station are approximately $45^{\circ}$, irrespective of the constellation size. Similar squint angle limits are realistic for other spaceborne RF systems; see e.g., [38], [39]. Consequently, the implicit assumption that our phased array maintains a coherent RF beam for the range of expected 


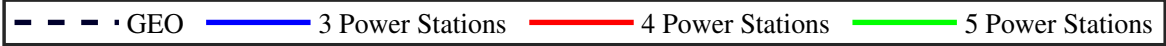
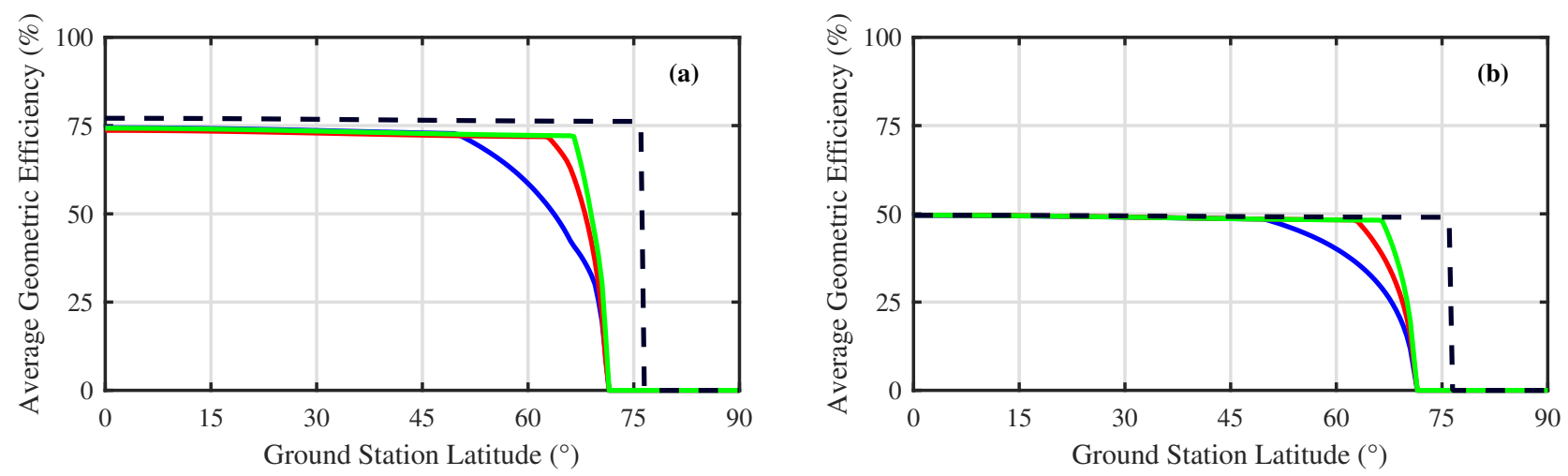

Fig. 8. Sensitivity of day-averaged geometric efficiency $\bar{G}_{d}^{*}$ with latitude for a constellation of dual-sided power stations in a $20,184 \mathrm{~km}$ circular equatorial MEO with (a) $\delta_{h}=5^{\circ}$ and (b) $\delta_{h}=45^{\circ}$.

TABLE I

Day-AVERAged GeOMETRic EFFiciencies For Ground Stations LOCATED AT $33^{\circ}$ LATITUDE WITH $\delta_{h}=5^{\circ}$

\begin{tabular}{cccc}
\hline \multicolumn{4}{c}{ Day-Averaged Geometric Efficiency, $\bar{G}_{d}^{*}(\%)$} \\
$N=3$ & $N=4$ & $N=5$ & GEO \\
\hline 73.5 & 72.8 & 73.3 & 76.7 \\
\hline
\end{tabular}

squint angles is also realistic. Moreover, note that the results in Fig. 8 are independent of each ground station's footprint size. Practical constraints on footprint size may further constrain the minimum allowable elevation angle, thereby reducing the maximum possible ground station latitude. Footprint sizing is incorporated separately into the LCOE model in Sec. IV

The remaining sections of this paper develop the LCOE model and apply it to several case studies that leverage the results in Fig. 8. These case studies assume a ground station latitude of $33^{\circ}$ and that $\delta_{h} \leqslant \delta_{t}$ (to guarantee persistent ground station coverage). As a result, the minimum elevation angles for the three, four, and five power station constellations are approximately $11^{\circ}, 24^{\circ}$, and $31^{\circ}$; see Fig. 5. Table II summarizes the day-averaged geometric efficiencies $\bar{G}_{d}^{*}$ for ground stations at $33^{\circ}$ latitude.

Surprisingly, Table II shows that the three power station constellation has a higher value of $\bar{G}_{d}^{*}$ than the four and five power station constellations. The expected result is a small increase in $\bar{G}_{d}^{*}$ as the constellation size increases. This discrepancy is due to our decision to use a non-optimal criterion for assigning power stations to ground stations. Whereas our assignment criterion guarantees equal coverage of each ground station by each power station, it does not guarantee that the hand-offs between power stations result in the same or higher instantaneous power transmission. Therefore, our assignment criterion forces hand-offs that sometimes reduce instantaneous power transmission.

Achieving the expected result would require implementing a new assignment criterion based on power transmission where different ground stations would realize different coverage levels and geometric efficiencies. Changing ground station coverage would also change the transition elevation angles, thereby affecting ground station footprint size. Hence, an alternative assignment criterion may leverage the LCOE instead of power transmission to optimally account for elevation angle effects on the size of each ground station's footprint.

\section{System Modeling And Figure of Merit}

In this section, we describe the system model developed for the Caltech SSPP architecture [14] and discuss how it is used to compare different system instantiations. While this model is specific to the Caltech SSPP concept, it can easily be generalized to model other concepts.

\section{A. Overview of System Model}

The system model [14] uses parameters from the Caltech SSPP to estimate the Levelized Cost of Electricity (LCOE), i.e., the price at which a space solar power system can sell electricity. LCOE provides a meaningful figure of merit for assessing different system instantiations. We are looking for those instantiations that lead to the lowest LCOE. While LCOE varies greatly across the globe from around $\$ 0.05 / \mathrm{kWh}$ to over $\$ 0.50 / \mathrm{kWh}$ and can even be as high as $\$ 15 / \mathrm{kWh}$ for expeditionary forces in military or humanitarian relief operations [40], the relative value of one system instantiation versus another can be established by comparing their predicted LCOEs. As we note later on, the predicted LCOEs are based on an uncalibrated cost model for the space vehicles, and thus are likely not good estimates of the actual cost of electricity from any system instantiation. However, the LCOEs do provide a valid relative figure of merit between different system instantiations because the systems are assessed with the same metric.

The space vehicle mass is estimated off-line using a mass model developed from [16], [30], [31]. The mass model currently estimates the areal mass density of the tiles. This areal density and the deployed aperture area determine the mass of the aperture. To simplify the mass model, the masses of the payload structure (including the booms, longerons, 
and battens), deployment mechanism, and spacecraft bus are carried in the model as fixed parameters.

The model imposes mass and volume constraints consistent with the launch system's payload mass, payload fairing, and the stowed volume of the space vehicles. Referring to Sec. II. a $60 \mathrm{~m} \times 60 \mathrm{~m}$ space vehicle packages into a cylindrical stowed configuration approximately $2.2 \mathrm{~m}$ in diameter and $1.8 \mathrm{~m}$ in height. As an example, a SpaceX Falcon Heavy has sufficient volume to launch 15 stowed space vehicles inside its $5 \mathrm{~m}$ diameter payload fairing while maintaining a $10 \%$ volume reserve for supporting structure [41]. If we take the mass-to-orbit for the Falcon Heavy (13 MT) less a 3\% launch reserve, we find that the Falcon Heavy is volume-limited if the space vehicle mass does not exceed $840 \mathrm{~kg}$, i.e., the payload fairing volume becomes the limiting factor in determining how many space vehicles can be accommodated on a single launch. Likewise, if the space vehicle mass exceeds $840 \mathrm{~kg}$, the Falcon Heavy is mass-limited, meaning we must decrease the number of satellites per launch.

The input that scales the space solar power system system is the average power provided to an electrical grid. This value, various efficiencies, and the aperture size determine how many space vehicles per power station are required in a given orbit to achieve the required power. The number of space vehicles determines the number of launches required for a given launch system. The cost for the space segment is then computed using one of the aforementioned cost models to obtain the first unit cost and a learning curve [42] to compute production costs for the space vehicles and launchers. Using the service life, we then compute the total energy collected and divide that into the total space segment cost to estimate the space segment LCOE.

For simplicity, the current instantiation of the system model does not account for on-orbit space vehicle failures. While replacing a failed space vehicle increases the overall cost, and hence, LCOE, it is difficult to estimate the impact of one or more space vehicle failures on LCOE for two reasons. First, at our concept's current level of maturity, it is difficult to ascertain a realistic space vehicle failure rate. Second, it is unclear how many space vehicle failures a power station can tolerate before its operational capability degrades to the point beyond which it can no longer profitably provide power to the grid. We can conceivably demand that all the space vehicles in a power station are always operational, but this is overly conservative, and consequently, drives up LCOE. Alternatively, we can set a threshold, e.g., no less than $90 \%$ of the space vehicles in a power station must be operational at any given time, beyond which replacements are launched, but the basis for this decision combines systems engineering with the economics of energy production and falls outside the scope of our current work.

We address the ground segment in a similar fashion. We assume the radiated RF energy leaving the aperture is coherent and can either be focused (Fresnel zone case) or radiates as a plane wave (far-field case) to the Earth. We compute the ground footprint of the main lobe based on its radius from the maximum to the first null and estimate that $80-84 \%$ of the radiated energy resides in the main lobe [43]. The model accounts for land costs and estimates the costs of the rectennas and the construction of infrastructure. We then compute the LCOE for this segment by dividing the power provided to the grid by the ground segment cost. An estimate for off-line energy storage is also included in the ground segment cost as the proposed space solar power system is designed to deliver an average power to the grid. The sum of the space and ground segment LCOEs gives the system LCOE.

Lastly, the system model contains allocations for recurring operations and maintenance costs, along with margin to account for costs not currently modeled, e.g., loan repayments. These costs are folded into the individual LCOE contributions.

\section{B. Space Segment Modeling}

We model the space segment performance using a link budget. Cost is determined by computing the number of satellites and launches needed to meet a requirement for power delivered to the grid. The parametric cost model also includes an estimate of the development cost of the space vehicle (spacecraft and payload) which is added into the space system cost as a one-time charge. Finally, the LCOE contribution of the space segment is computed using the total energy delivered to the grid over the service life of the space segment divided by the total cost of the space segment.

The link budget relates the solar energy collected in space to the energy received on the ground. From [14], the received power on the ground, $P_{r}$, is

$$
P_{r}=\left[\eta_{\mathrm{PV}} \eta_{\mathrm{DC}-\mathrm{RF}} \eta_{\mathrm{Tx}} \eta_{\mathrm{dif}} \bar{G}_{d}^{*} A_{\mathrm{PV}} \mathrm{AM} 0\right]\left(\frac{f}{c \rho}\right)^{2} A_{\mathrm{PV}} A_{r}
$$

where $\eta_{\text {dif }}$ is the percentage of radiated power contained in the main lobe of the RF beam, $A_{r}$ is the area of the main lobe projected onto Earth, $f$ is the transmitting frequency, and $c$ is the speed of light. The day-averaged geometric efficiency $\bar{G}_{d}^{*}$ is treated as another reduction factor in the link budget. The effect of including $\bar{G}_{d}^{*}$ is that the number of satellites per power station is increased to ensure that the system can deliver power $P_{g}$ on average. This increase results in periods of time where the power station delivers more than $P_{g}$ to the grid, and periods of time where it delivers less than $P_{g}$, driving a requirement for offline energy storage at the ground site to manage the power delivered to the grid. We return to this later. Note that the link budget neglects atmospheric losses because the associated reduction factor for our chosen $10 \mathrm{GHz}$ operating frequency is small compared to the other reduction factors in (22).

In the Caltech SSPP architecture, $A_{\mathrm{PV}}$ is the same as the area of the transmitting antenna array. The most efficient use of the beamed power comes from sizing the ground rectenna array to fill the projected area of the main lobe from the power station. We assume that the angle subtended by the main lobe can be estimated by the diffraction limit relationship

$$
\theta_{\mathrm{ML}}=k \frac{c}{f \sqrt{A_{\mathrm{PV}}}}
$$

where $k \approx 1$. Hence, the projected area is approximately

$$
A_{r}=\frac{c^{2} \rho^{2}}{f^{2} A_{\mathrm{PV}}} \text {. }
$$


By introducing the efficiency of the rectenna, $\eta_{\text {rect }}$, and the DC-to-AC conversion efficiency, $\eta_{\text {DC-AC }}$, we can compute the power delivered to the grid:

$$
P_{g}=H_{s} H_{g} \text { AM0 } A_{\mathrm{PV}}
$$

where $H_{s}=\eta_{\mathrm{PV}} \eta_{\mathrm{DC}-\mathrm{RF}} \eta_{\mathrm{Tx}} \eta_{\mathrm{dif}} \bar{G}_{d}^{*}$ and $H_{g}=\eta_{\text {rect }} \eta_{\mathrm{DC}-\mathrm{AC}}$.

If $P_{g}$ is the total power demand for the grid, then 25 yields the total PV area required on-orbit. If each satellite in a power station has a PV area $A_{s}$ and the power station functions as a very large phased array, then we can compute the required number of satellites, $N_{s}$, needed to provide $P_{g}$ to the grid, as follows:

$$
N_{s}=\left\lceil\frac{A_{\mathrm{PV}}}{A_{s}}\right\rceil
$$

where the brackets in 26 indicate rounding up of a fractional part to the nearest integer, reflecting that we cannot provide a fraction of a satellite.

The number of satellites per launch vehicle, $N_{\mathrm{LV}}$, is similarly computed by using the launcher's mass-to-orbit, $M_{\text {orb }}$, derated by a factor $\kappa(0<\kappa<1)$ to allow for launch margin, divided by the space vehicle mass, $m_{s}$ :

$$
N_{\mathrm{LV}}=\left\lceil\frac{\kappa M_{\mathrm{orb}}}{m_{s}}\right\rceil \text {. }
$$

We can now compute the cost of the space segment. We establish the first unit cost, denoted T1, and then generate the costs for all $N_{s}$ satellites using a learning curve. For what follows, we use a parametric cost model [44] which models the payload cost as

$$
C_{\mathrm{PL}}(\$ \mathrm{M})=a_{1} m_{\mathrm{PL}}^{b_{1}}(\mathrm{~kg})
$$

where $a_{1}=0.29725$ and $b_{1}=0.7$. Similarly, the spacecraft cost (bus without payload) is modeled as:

$$
C_{\mathrm{SV}}(\$ \mathrm{M})=a_{2} m_{\mathrm{SV}}^{b_{2}}(\mathrm{~kg})
$$

where $a_{2}=0.4461$ and $b_{2}=0.662$. The total cost $\mathrm{T} 1$ is simply the sum of (28) and 29). We also estimate development costs parametrically; these costs are added to the total cost after applying a learning curve to the satellite production run. Finally, the cost of the launch system is computed using a learning curve for the vehicle's production run.

The $\mathrm{T} 1$ costs are fed into a univariate log-linear learning curve model that depends on the rate of learning, $L$. The learning curve then uses the $\mathrm{T} 1$ costs and a lot production size to compute the costs of subsequent lots, $C_{i}$, according to the formula [42]:

$$
C_{i}=\mathrm{T} 1(i)^{\frac{\log (L)}{\log (2)}}
$$

where in this context $i$ denotes the lot number. Learning in production typically results in decreasing production times and costs as the production line learns to become more efficient through repetition. Learning generally stops when the cost of a lot is about $50 \%$ of the $\mathrm{T} 1$ costs.

The learning curve effect is sensitive to how lot size is defined. For instance, if 100 units of a product are to be produced and the T1 cost is $\$ 100$, the average cost per unit grows with the number of units per lot. Increasing the lot size implies parallel lines of production where each line learns
TABLE II

IMPACT of Lot SizE on Average Unit Cost

\begin{tabular}{cc}
\hline Lot Size & Unit Cost \\
\hline 1 & $\$ 52.41$ \\
2 & $\$ 54.81$ \\
5 & $\$ 62.03$ \\
\hline
\end{tabular}

independently from the others. Table III shows the impact to average cost for a learning rate of $85 \%$ for lot sizes of 1,2 , and 5 units. The table shows that a single production line achieves a lower average cost over the production run than parallel lines of production. What is not captured in this model, however, is the time to market impact of a single production line versus multiple production lines. In the current context, a single production line requires more time than multiple production lines to build all the required satellites, hence delaying the time to initial operational capability. Our model allows the user to supply a lot size for the production of satellites and launch systems. The financial penalties (e.g., longer time for return on investment, and hence longer time to profitability) possibly incurred with small lot sizes are not taken into account in the current version of the model.

\section{Ground Section Modeling}

Ground segment modeling is mainly aimed at developing cost estimates for this segment. The two efficiencies associated with the ground, $\eta_{\text {rect }}$ and $\eta_{\text {DC-AC }}$, were used earlier in the link budget to compute the number of satellites required to deliver an average power $P_{g}$ to the grid. Thus, the focus for this part of the system model is establishing the ground segment's contribution to the LCOE.

Equation (24) provides an estimate for the area required to capture the main lobe of the RF beam. The main lobe contains $84 \%$ of the radiated energy. Equation (24) is an excellent approximation for the case when the power station is directly overhead the ground station, i.e., at a $90^{\circ}$ elevation angle relative to the ground.

For the equatorial MEO considered here, the projected area of the main lobe transmitted from each power station changes with time. At low elevation angles, the projected area is elongated along the line of sight from the power station to the ground station and broadened in the direction perpendicular to the line of sight. At high elevation angles, the elongation is minimized and the projection approaches a circle. For a ground station with a given latitude and longitude, the projected area is smallest at the maximum elevation angle which occurs when the power station is at the same longitude as the ground station. A ground station for MEO is necessarily larger than that for GEO due to the relative motion of the power station to the ground. We assume that the cost per square meter of land for a ground station is independent of both its geographical location and the power station's orbit. These assumptions allow us to easily assess how the size of the ground station's footprint impacts ground segment costs.

Consider the GEO case where the ground station is at the same longitude as the power station but at a latitude $\phi$ where 
$0^{\circ}<\phi<90^{\circ}$. The slant range, $\rho$, to the ground station is given by (8) where $\Omega=\phi$. Along the direction of $\rho$, the footprint of the beam is elongated by Earth's curvature. The elongation can be computed simply by finding the points where the main lobe of the RF beam (assumed to have a beamwidth of $\theta_{\text {ML }}$ given by (23) ) intercepts the Earth's surface. After some trigonometry, we find that the main lobe subtends the angle $\Delta \phi$ relative to the Earth's center, given by:

$$
\begin{aligned}
\Delta \phi= & \sin ^{-1}\left(\frac{\rho_{+}}{R_{\oplus}} \sin \left(\psi+\frac{\theta_{\mathrm{ML}}}{2}\right)\right) \\
& -\sin ^{-1}\left(\frac{\rho_{-}}{R_{\oplus}} \sin \left(\psi-\frac{\theta_{\mathrm{ML}}}{2}\right)\right)
\end{aligned}
$$

where

$$
\begin{gathered}
\psi=\sin ^{-1}\left(\frac{R_{\oplus}}{\rho} \sin (\phi)\right), \\
\rho_{ \pm}=r \cos \left(\psi_{ \pm} \pm \frac{\theta_{\mathrm{ML}}}{2}\right) \\
\pm \sqrt{\left(r \cos \left(\psi_{ \pm} \pm \frac{\theta_{\mathrm{ML}}}{2}\right)\right)^{2}+R_{\oplus}^{2}-r^{2}},
\end{gathered}
$$

and $\psi_{ \pm}=\psi \pm \theta_{\mathrm{ML}} / 2$.

The main lobe projects an ellipse with semimajor axis $A_{\text {maj }}=R_{\oplus} \Delta \phi / 2$ and semiminor axis approximately given by $B_{\text {min }}=R_{\oplus} \theta_{\mathrm{ML}} / 2$. The resulting projected area is then

$$
A_{r}=\frac{\pi}{4} A_{\text {maj }} B_{\text {min }} .
$$

Note that as $\phi \rightarrow 0, \psi \rightarrow 0$ and $A_{\text {maj }}=B_{\text {min }}$, as expected.

Computing the maximum ground station area for MEObased systems is more complicated, and hence is calculated with Analytical Graphics, Inc.'s Systems Tool Kit ${ }^{\mathrm{TM}}$ (STK) [45].

Given the ground station footprints, we can determine the cost for building each ground station. We assume that the receiving elements are rectennas [46] so that each element receives radio frequency power and converts it to DC electrical power. The rectifying aspect of the rectenna eliminates the need to phase control the receive antenna array, greatly simplifying the ground system. We further assume the rectennas are spaced in half-wavelength intervals to capture all the energy in the main lobe. The rectenna spacing combined with the ground station area gives the number of required rectennas.

Along with the cost of land and rectennas, the model includes costs for construction, operations and maintenance, and off-line energy storage. As mentioned above, the geometric efficiency for both MEO and GEO imposes the need to store energy in excess of the grid requirements for times when the power station is providing less energy than required. The cost of energy storage is based on Tesla's utility level battery installation [47], the geometric efficiency, and the specified $P_{g}$ for the system. The storage scales with the specified $P_{g}$.

We now turn to the analysis and comparison of a GEObased space solar power architecture with a MEO-based one.
TABLE III

PARAMETERS FOR GEO AND MEO ARCHITECTURES

\begin{tabular}{l|c}
\hline \multicolumn{1}{c|}{ Parameter } & Value \\
\hline Power to grid & $100 \mathrm{MW}$ \\
Power transmission frequency & $10 \mathrm{GHz}$ \\
Spacecraft areal density & $0.505 \mathrm{~kg} / \mathrm{m}^{2}$ \\
Spacecraft area & $3,600 \mathrm{~m}^{2}$ \\
Ground station latitude & $33^{\circ}$ \\
\hline Efficiencies & \\
\hline PV $\left(\eta_{\mathrm{PV}}\right)$ & $17 \%$ \\
DC-to-RF $\left(\eta_{\text {DC-RF }}\right)$ & $50 \%$ \\
Transmit $\left(\eta_{\text {Tx }}\right)$ & $100 \%$ \\
Diffraction $\left(\eta_{\text {dif }}\right)$ & $84 \%$ \\
RF-to-DC $\left(\eta_{\text {RF-DC }}\right)$ & $82 \%$ \\
DC-to-AC $\left(\eta_{\text {DC-AC }}\right)$ & $95 \%$ \\
Geometric - GEO & See Table $\mathrm{I}$ \\
Geometric - MEO & See Table \\
\hline Service life & 15 years \\
Launch vehicle & Falcon Heavy \\
Learning rate & $85 \%$ \\
\hline Spacecraft dimensions (stowed) & \\
\hline Diameter & $2.2 \mathrm{~m}$ \\
Height & $1.8 \mathrm{~m}$ \\
\hline
\end{tabular}

\section{ARChitecture COMPARISONS}

Consider the problem of providing an average power of $100 \mathrm{MW}$ to an electrical grid. In the United States, this amount of power would supply between 62,000 and 145,000 homes, depending on the state [48]. Due to the landmass distribution in the Northern Hemisphere, we assume a ground station latitude of $33^{\circ} \mathrm{N}$. At this latitude, a power station in an equatorial orbit can provide electricity to many of the world's large population centers. For both cases, we assume a Falcon Heavy launch vehicle and impose fairing volume constraints on the number of satellites per launcher. We chose the Falcon Heavy due to its large lift capacity to GEO [49] and its estimated lift capacity to MEO. Other commercially available launch systems have a higher cost per kilogram delivered to GEO. The input parameters for both cases are identical except for the orbital altitudes and geometric efficiencies from Table I The parameters for the model are listed in Table III

The parameters in Table III correspond to a power station using perovskite PV cells. Perovskites [50] are an emerging PV technology and hold the promise of being manufactured at relatively low cost because they are fabricated using a wet chemical process rather than a crystal or epitaxial growth process. However, their overall device efficiency is lower than even modestly performing silicon PV cells. Based on published data and ongoing research at Caltech, we project a $17 \%$ efficiency for perovskite PV cells. Another attractive characteristic of perovskites is their tolerance to ionizing radiation. Tests done at Caltech [51] and elsewhere [52], [53] have shown that while perovskites suffer some displacement damage in high radiation fields, the damage can be annealed out to return the device to its undamaged operating state. This eliminates the need for cover glass, greatly reducing the mass 
of the cells.

Each perovskite cell is 1-2 $\mu \mathrm{m}$ thick. When we consider the areal mass density of the PV material, integrated circuit and routing plane, antenna planes, and carbon fiber support structure, we arrive at an estimated tile areal mass density of $0.505 \mathrm{~kg} / \mathrm{m}^{2}$. There are separate mass allocations in the model for the spacecraft bus and the booms, longerons, and battens [16], [30], [31] that form the supporting structure for the tiles. Currently, we allocate $12 \mathrm{~kg}$ and $80 \mathrm{~kg}$ for the booms and longerons/battens, respectively, along with $30 \mathrm{~kg}$ for the deployment mechanism and $80 \mathrm{~kg}$ for the spacecraft bus. The latter is a rough estimate based on the current state-of-the-art in small satellite technology. Based on this mass model, we arrive at a total mass of 2,020 $\mathrm{kg}$ per space vehicle.

We have also modeled a system using GaAs PV cells fabricated using an epitaxial lift-off process with a $25 \%$ efficiency. These cells require a cover glass [54] and are somewhat thicker than the perovskites, resulting in an areal mass density of $0.809 \mathrm{~kg} / \mathrm{m}^{2}-$ a $60 \%$ increase in mass over the perovskite system for at most a $10 \%$ increase in PV efficiency. This leads to a significant decrease in overall RF specific power. While the resulting LCOE for GaAs-based systems can be lower than perovskite-based systems, the low tolerance of GaAs cells to radiation makes them less attractive for long space missions, especially in the MEO environment.

Not included in Table III are estimates for operations and maintenance costs and margin. Operations and maintenance costs are assumed to be $\$ 400 \mathrm{M}$ per year per power station, and a $20 \%$ margin is added to the "raw" LCOEs. The operations and maintenance allocation is sufficient to cover the cost of launch insurance, which in the event of launch failure reimburses the cost of a launch vehicle and its payloads. Launch insurance typically costs approximately $4 \%$ of the value of the launch vehicle and its payloads. At present, a launch failure rate of $2 \%$ is assumed for the Falcon Heavy, which is reasonable considering the current launch failure rates of some of its contemporaries, e.g., the Atlas V and Falcon 9. This amounts to launch insurance costs of less than $\$ 4.3 \mathrm{~B}$ over the 15 year system lifetime for the five power station MEO architecture (Sec. V-B), something easily accommodated by the total operations and maintenance allocation in the model.

\section{A. GEO Architecture}

For the GEO case, we assume an equatorial geosynchronous orbit with a ground station at a latitude of $33^{\circ} \mathrm{N}$ and the same longitude as the power station. The transmitted power is the same for ground stations located at the same latitude on either side of the equator, but there is more landmass above the equator at higher latitudes than below. We assume a unique one-to-one correspondence between the number of power stations and ground stations, i.e., power is beamed to only one point on the ground from any given power station.

Fig. 9 provides a breakdown of the major cost contributors to the LCOE for a single GEO power station. The estimated LCOE for this system is $\$ 2.32 / \mathrm{kWh}$. Fig. 9 shows that $69 \%$ of the LCOE is due to the cost of the space vehicles. In this architecture, there are 505 satellites with a mass of $2,020 \mathrm{~kg}$

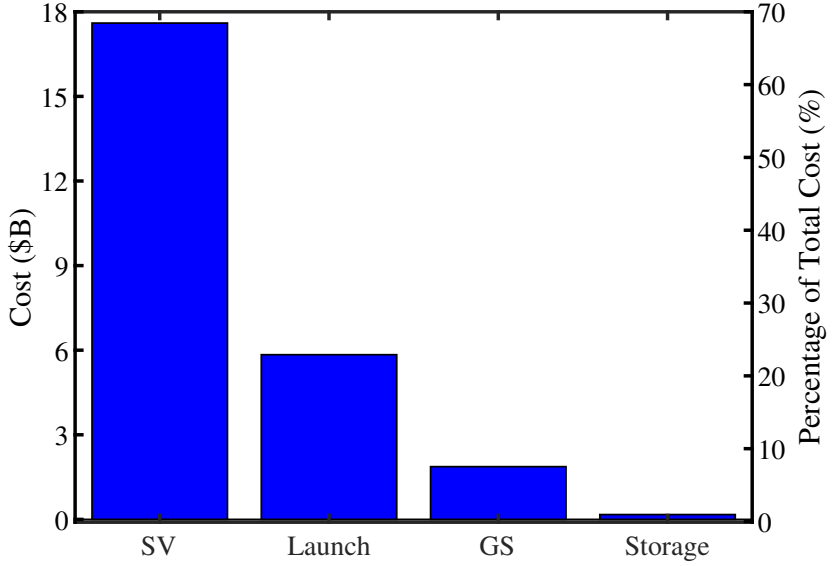

Fig. 9. LCOE breakdown for one GEO power station and ground component. SV and GS denote space vehicles and ground segment, respectively. Most of the cost resides in the space vehicles.

each at a T1 cost of $\$ 68 \mathrm{M}$. The second largest contributor to the LCOE is the launch cost at $23 \%$. We employed the Falcon Heavy with the $5 \mathrm{~m}$ fairing at a T1 cost of $\$ 130 \mathrm{M}$ and derated the mass-to-GEO capability by $3 \%$ to allow for required launch margin. The $5 \mathrm{~m}$ fairing provides sufficient volume for up to 15 satellites. However, given the mass of the satellites, the Falcon Heavy can only carry 6 satellites to GEO per launch. Thus, 85 launches are required.

The MEO architecture (Sec. V-B consists of three, four, or five orbiting power stations with the corresponding number of ground stations receiving the beamed power. In order to provide a direct comparison between the GEO and MEO architectures, we compute the LCOEs for three, four, and five GEO power stations with the corresponding number of ground stations. Table IV summarizes these results. The LCOE is weakly dependent on the number of power stations in GEO. The change in LCOE from a single power station to three or more power stations is due to learning effects. With three or more power stations, most of the space vehicles are produced at half the T1 cost. Since the average cost per vehicle asymptotically approaches $\mathrm{T} 1 / 2$ as the number of space vehicles produced increases, there is no significant difference in the space segment LCOEs with three or more power stations. In short, building more power stations allows greater amortization of the development costs, which are independent of the number of power stations built. This results in a small decrease in the LCOE from the single power station case.

We now move to the MEO architecture. We will compare three different variants of the MEO architecture with each other and the GEO architecture.

\section{B. MEO Architecture}

Unlike the GEO architecture, where one power station services one ground station, MEO architectures require multiple power stations to guarantee that a single ground station receives an (almost) uninterrupted flow of energy from space. An uninterrupted flow of energy requires a minimum of three power stations phased $120^{\circ}$ apart in true anomaly in a nominal 12 hour orbit. For any given ground station, two power stations 
TABLE IV

INDEPENDENCE OF LCOE WITH NUMBER OF POWER STATIONS FOR THE GEO ARCHITECTURE

\begin{tabular}{c|c|c|c|c|c}
\hline \multirow{2}{*}{ \# of Power Stations } & \multicolumn{3}{|c|}{ LCOE Contributions $(\$ / \mathrm{kW} \mathrm{h})$} & \multirow{2}{*}{ LCOE $(\$ / \mathrm{kW} \mathrm{h})$} \\
\cline { 2 - 5 } & Space Vehicles & Launch & Ground Segment & Storage & \\
\hline 1 & 1.60 & 0.53 & 0.17 & 0.02 & 2.32 \\
3 & 1.58 & 0.53 & 0.17 & 0.02 & 2.30 \\
4 & 1.58 & 0.53 & 0.17 & 0.02 & 2.30 \\
5 & 1.58 & 0.53 & 0.17 & 0.02 & 2.30 \\
\hline
\end{tabular}

are out of view most of the time; the out-of-view power stations service other ground stations. The architecture then leads to one ground station for each orbiting power station with ground station spacing in longitude equivalent to the true anomaly spacing of the power stations. Unlike the GEO architecture, each power station provides energy at some point in its orbit to every ground station.

We investigate the LCOEs for three, four, and five power station constellations, all in an equatorial MEO at an altitude of $20,184 \mathrm{~km}$. All the ground stations are located at $33^{\circ} \mathrm{N}$ latitude and have the same spacing in longitude as the power stations have in true anomaly. This can result in ground stations located in the ocean. We have also considered cases that adjust the ground stations to nearby landmasses when they end up in the ocean. This changes handover conditions (discussed below), but the changes are not significant enough to alter the conclusions drawn from the equally-spaced ground station case.

The three power station constellation with power stations spaced $120^{\circ}$ apart in true anomaly is shown in Fig. 10 A power station begins transmitting to a ground station at an elevation angle of $11.2^{\circ}$ when rising (relative to the ground station) and ceases transmitting at the same elevation angle when setting. At this transition elevation angle, the power beaming to the ground station is handed off to the rising power station, as depicted in Fig. 10 The setting power station then provides power to the ground station to the east.

The low elevation angle drives the ground area required to capture the main lobe of the radiated RF beam. For nonequatorial ground stations, the ellipse formed by the projection of the main lobe on the ground rotates in azimuth relative to North and changes size as the power station transits from rising to setting over the ground station. Thus, the receiving rectennas must cover the entire area swept out by the projection of the main lobe which is large at low elevation angles.

The four power station constellation (Fig. 11) spaces the power stations $90^{\circ}$ apart in true anomaly. The minimum elevation angle increases to $23.7^{\circ}$. This results in a factor of 3.4 reduction in ground station area from the three power station case and an equivalent reduction in cost per ground station.

The five power station constellation (Fig. 12) decreases the phasing to $72^{\circ}$ in true anomaly with a minimum elevation angle of $30.8^{\circ}$. The larger minimum elevation angle results in ground stations that are a factor of 6.15 times smaller than the three power station case and 1.8 times smaller than the four power station case. We anticipate that the cost per ground station will decrease by about the same factor.

To better illustrate how the ground station areas are driven by the number of power stations, we used $\mathrm{STK}^{\mathrm{TM}}$ to project the main lobe footprint around a ground station for rising and setting power stations. Figs. $13 \mathrm{a}, 13 \mathrm{~b}$, and $13 \mathrm{c}$ show the footprints for the three, four, and five power station cases, respectively.

For each constellation, the number of space vehicles per power station is fixed by the on-orbit PV area required to provide an average power to the grid, in this case $100 \mathrm{MW}$, after accounting for the reduction factors in the link budget. Table V compares the number of space vehicles (denoted SVs) per power station (denoted PS) for the three MEO architectures and the GEO architecture required to deliver $100 \mathrm{MW}$ to the grid. The number of space vehicles per power station in MEO is largely determined by the day-averaged geometric efficiency (Table II); hence, each MEO architecture requires roughly the same number of space vehicles. Since the same launch vehicle (Falcon Heavy) can lift more mass to MEO than GEO, each launch delivers 7 satellites to MEO, compared to only 6 in GEO. This significantly decreases the LCOE contribution attributed to the launch costs. Note that we assume that a single launch vehicle may deliver space vehicles destined to multiple power stations in a constellation. This is operationally more cost effective because it allows us to always launch full or nearly full payloads of space vehicles, but it carries the caveat that some space vehicles may need to re-phase after launch.

Next, Table VI compares the results for the three MEO constellations and the baseline GEO system, each sized to provide an average of $100 \mathrm{MW}$ to a terrestrial power grid. From Table VI, the three power station constellation is the least cost effective of the MEO systems under consideration. The reason for this is the very large area required for each ground station. We assume that the cost per square meter for the ground segment is independent of power station size and the geographical location of the ground station. While the independence of the unit area cost of the ground segment from the space segment is reasonable, the assumption of the independence of cost from the location of the ground station is not. However, this assumption permits direct comparisons of the LCOE for different architectures since unit area ground costs, with some exceptions (e.g., prime land in Maui might go for $\$ 10,000 / a c r e$ [55] compared to Arizona desert land at $\$ 1,000 /$ acre [56]), do not vary enough to change the conclusions of the comparisons.

Fig. 14 shows how the LCOE and the the required ground station area change with constellation size. The LCOE dramatically decreases as the number of power stations increases from three to four due to the large reduction in ground station 


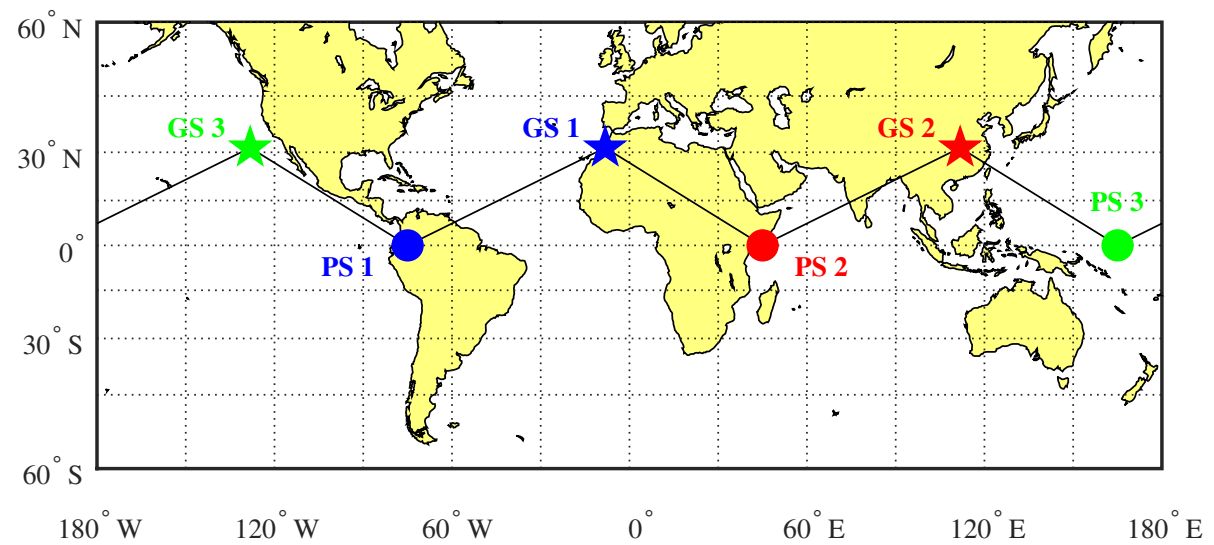

Fig. 10. Three power stations with $120^{\circ}$ phasing ensures 24 hour power beaming to a given ground station. PS and GS refer to power stations and ground stations, respectively.

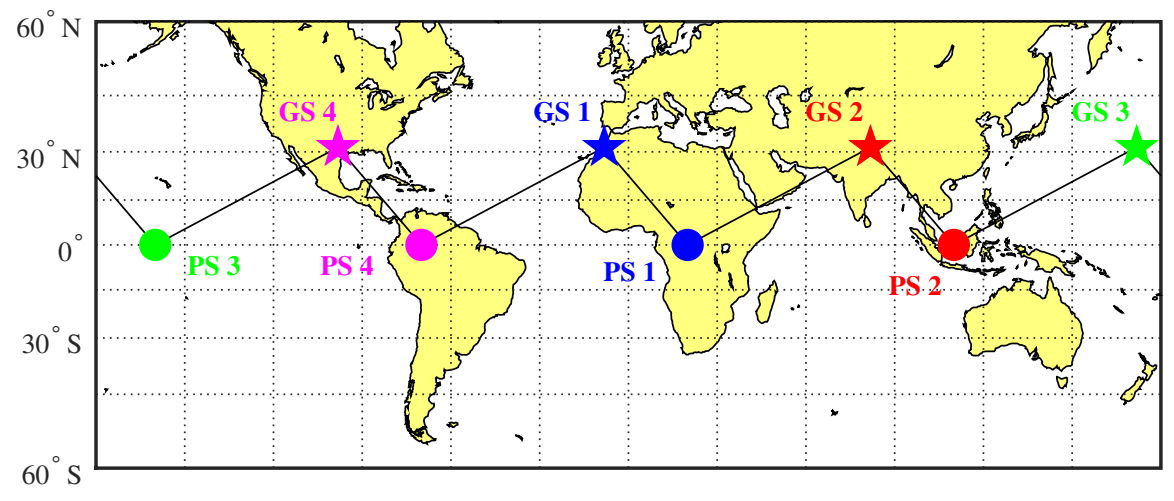
$180^{\circ} \mathrm{W}$
$120^{\circ} \mathrm{W}$
$60^{\circ} \mathrm{W}$
$60^{\circ} \mathrm{E}$
$120^{\circ} \mathrm{E}$
$180^{\circ} \mathrm{E}$

Fig. 11. Four power station system with $90^{\circ}$ phasing. The Pacific Ocean ground station can be moved to Hawaii without loss of coverage or contact. PS and GS refer to power stations and ground stations, respectively.

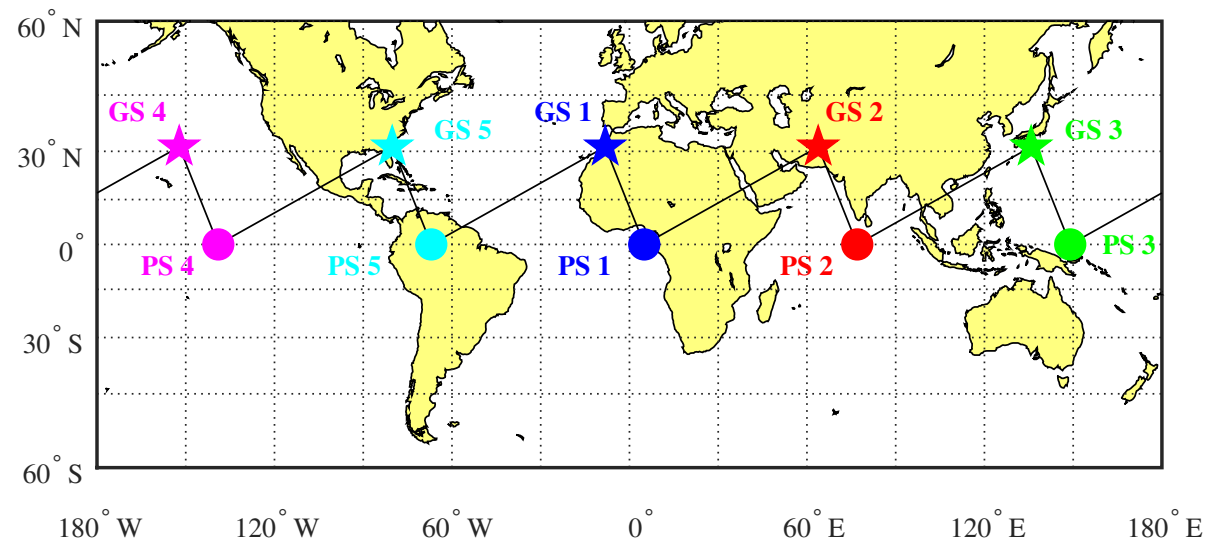

Fig. 12. Five ground stations equally-spaced at $72^{\circ}$ longitude and $33^{\circ}$ latitude. GS 3 and GS 4 can be moved to nearby landmasses without fundamentally changing the conclusions of our analysis. PS and GS refer to power stations and ground stations, respectively.

TABLE V

REQUiRED LAUNCHES FOR THE THREE MEO AND THE GEO ARCHITECTURES

\begin{tabular}{c|c|c|c|c}
\hline \# of Power Stations & \# of SVs/PS & Total \# of SVs & \# of Launches/PS & Total \# of Launches \\
\hline 3 & 524 & 1572 & 75 & 225 \\
4 & 529 & 2116 & 76 & 303 \\
5 & 526 & 2630 & 75 & 376 \\
GEO (1 PS) & 505 & 505 & 85 & 85 \\
\hline
\end{tabular}


TABLE VI

COMPARISONS OF THE THREE MEO AND THE GEO ARCHITECTURES

\begin{tabular}{l|c|c|c|c|c}
\hline \multirow{2}{*}{ Parameters } & \multicolumn{3}{|c|}{ MEO Architectures } & \multirow{2}{*}{ GEO Architecture } \\
\cline { 2 - 4 } & $N=3$ & $N=4$ & $N=5$ & \\
\cline { 2 - 4 } & Altitude $(\mathrm{km})$ & 20,184 & 20,184 & 20,184 & 35,786 \\
& Phasing $\left(^{\circ}\right)$ & 120 & 90 & 72 & N/A \\
& Min. Elevation Angle $\left(^{\circ}\right)$ & 11.2 & 23.7 & 30.8 & 51.6 \\
& ${\text { Ground Area }\left(\mathrm{km}^{2}\right)}^{*}$ & 18.95 & 5.51 & 3.08 & 6.04 \\
\hline \multirow{3}{*}{$\operatorname{LCOE}(\$ / \mathrm{kWh})$} & Power Station & 1.65 & 1.66 & 1.65 & 1.60 \\
& Launch & 0.32 & 0.31 & 0.29 & 0.53 \\
& Ground Station & 0.41 & 0.23 & 0.20 & 0.17 \\
& Off-Line Storage & 0.01 & 0.01 & 0.01 & 0.02 \\
& Total LCOE & 2.39 & 2.21 & 2.15 & 2.32 \\
\hline
\end{tabular}
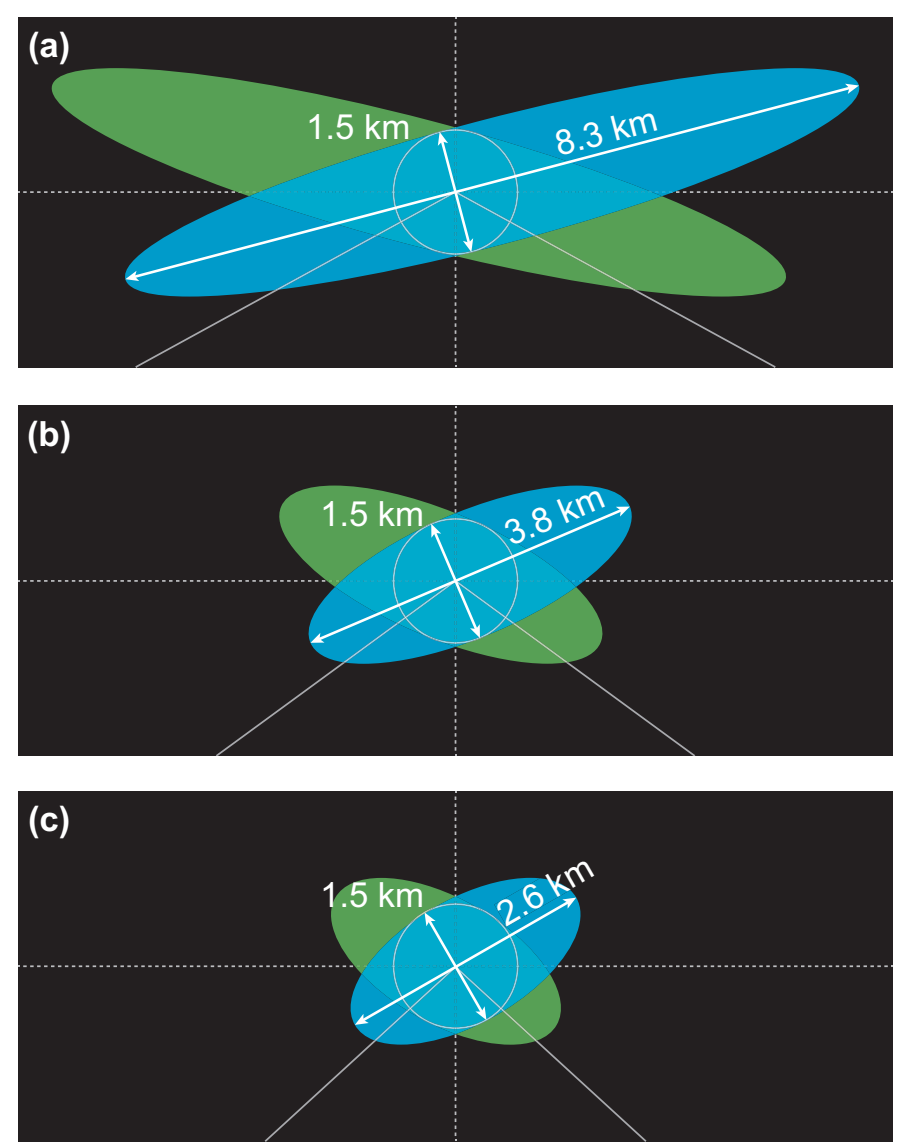

Fig. 13. Main lobe footprints at handovers for constellations with (a) three, (b) four, and (c) five power stations.

area. However, with four or more power stations, the LCOE is driven by the costs of the power stations and launch. Hence, the decrease in LCOE is modest as the number of power stations increases from four to five.

Per Table VI, the space segment (power stations plus launch) contributes between $\$ 1.94 / \mathrm{kWh}$ and $\$ 1.97 / \mathrm{kWh}$ to the LCOE for the three MEO architectures versus $\$ 2.13 / \mathrm{kWh}$ for the GEO architecture. The LCOEs of the MEO systems include increased ground costs and decreased off-line storage costs relative to the GEO system, the latter due to the shorter discharge periods experienced by the MEO systems. However,

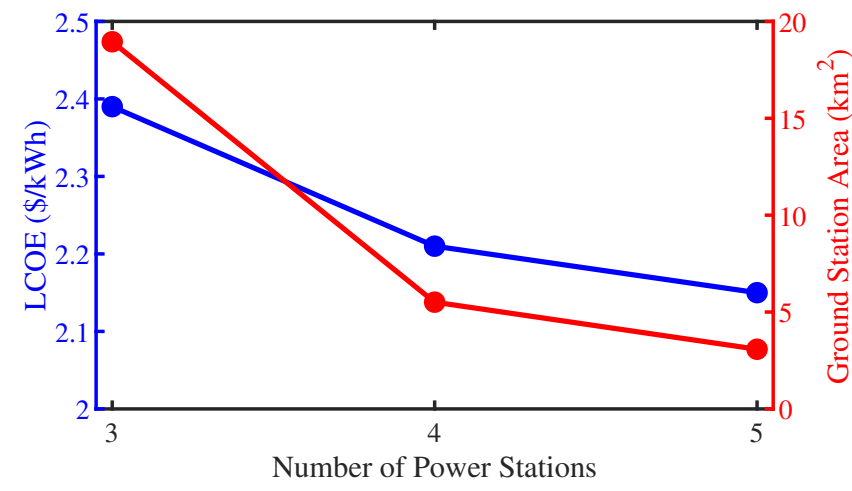

Fig. 14. The LCOE for the MEO constellations decreases rapidly due to the decrease in ground station area. The LCOE curve hints at asymptotic behavior as the costs are dominated by the space segment.

the GEO option assumes that a GEO orbital slot is available at the same longitude as the ground station. This is an optimistic assumption because the relevant GEO slots are currently occupied by other users [12]; vacant slots are largely located over the Pacific Ocean. As a result, a GEO power station is likely to transmit to a ground station located at a different longitude. This decreases the minimum elevation angle, thereby increasing the ground station area and cost. For example, moving the power station $63^{\circ}$ in longitude from a ground station at $33^{\circ}$ latitude increases the area of the ground station from $6.04 \mathrm{~km}^{2}$ to $8.4 \mathrm{~km}^{2}$. In turn, the LCOE contribution increases from $\$ 0.17 / \mathrm{kWh}$ to $\$ 0.19 / \mathrm{kWh}$. Thus, the LCOEs of the four and five power station MEO architectures are significantly lower than a GEO architecture where the power station is offset in longitude (a likely scenario) from its ground station.

Fig. 15 summarizes the relationship between the ground station footprint size and the LCOE for the MEO architectures. The three power station system's LCOE is larger than the LCOEs for the other two constellations because of its significantly larger ground station footprint. As the number of power stations increases, the ground segment's contribution to the LCOE decreases due to the rapid decline in the required ground station area. 


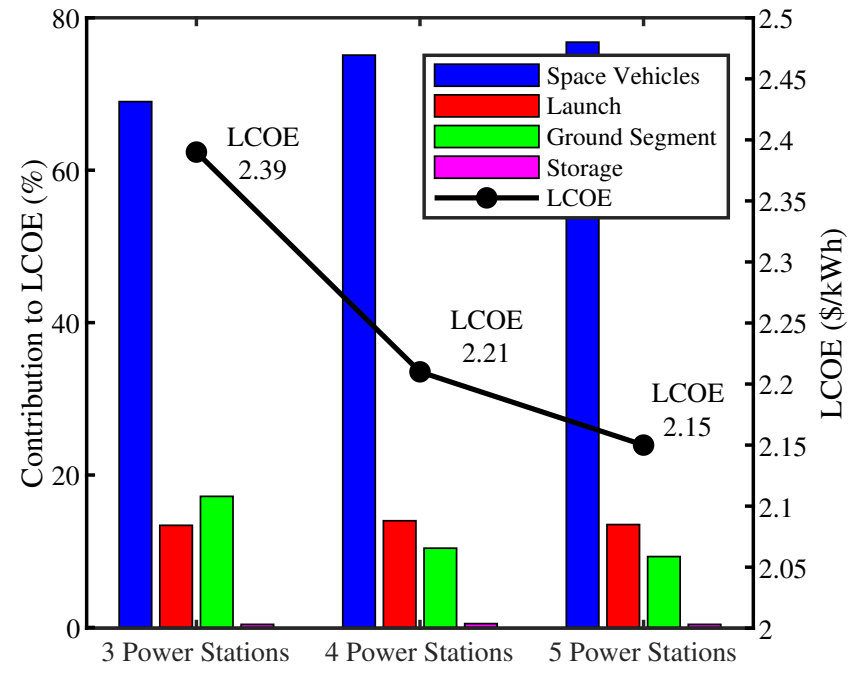

Fig. 15. The system LCOE is dominated by the cost of the space segment for MEO constellations.

\section{SUMMARY AND CONCLUSIONS}

We first extended the power-optimal guidance problem from [15] to accommodate constellations of space solar power stations transmitting to non-equatorial ground stations. We considered $60 \mathrm{~m} \times 60 \mathrm{~m}$ dual-sided power stations and showed that a MEO-based constellation of power stations has transmission performance comparable to a GEO system, but the constellation has the added capability of providing persistent (or nearly persistent) power to multiple terrestrial locations. The analysis showed that the transmission performance of a power station is sensitive to ground station latitude through the minimum allowable elevation angle. As the minimum allowable elevation angle decreases, the space segment can transmit the same amount of power to higher latitudes. However, as the latitude increases, so too does the ground station area required to capture the main lobe of the transmitted RF beam. The system analysis demonstrated that large ground station areas substantially increase a system's LCOE.

We then commenced the system analysis by considering a baseline GEO system delivering an average of $100 \mathrm{MW}$ of power to a terrestrial grid. This analysis resulted in an LCOE used for comparisons with the LCOEs generated for MEObased constellations. We applied power-optimal guidance to system modeling in order to assess the utility of MEO for space solar power. We described the system model developed for the Caltech SSPP, along with modifications made to accommodate MEO-based constellations. The analysis covered MEO constellations consisting of three, four, and five power stations with a one-to-one correspondence between the number of power and ground stations. Ground station areas were computed using STK $^{\mathrm{TM}}$ to project the main lobe of the transmitted power beam onto the ground at the minimum rising and setting elevation angles for each constellation. These areas drive the cost of the ground segment, which is folded into the costs used to determine the LCOE for each system. We then compared the LCOEs for the three MEO constellations and the baseline GEO system.

Our analysis showed that, counter to intuition, the three power station MEO architecture resulted in the highest LCOE of the analyzed architectures. This higher LCOE is directly traceable to the larger area required for each ground station, which in turn is due to the low elevation angles required to ensure each ground station receives continuous power. The four and five power station architectures had much lower LCOEs, equivalent to or better than the GEO architecture. We pointed out that the LCOE for GEO optimistically assumed that the power station was at the same longitude as the ground station. Relaxing this assumption resulted in a $\$ 0.13 / \mathrm{kWh}$ to $\$ 0.19 / \mathrm{kWh}$ difference between the GEO architecture and the four and five power station MEO architectures. Thus, the four and five power station MEO architectures offer a viable alternative to the GEO architecture because power can be provided at an LCOE equivalent to or better than GEO. This conclusion is subject to the caveat that we have neglected any possible increase in cost (and potentially mass) of the space vehicles due to hardening for the harsher radiation environment in MEO. However, with this in mind, our analysis suggests that MEO systems are competitive with GEO systems and may be more easily implemented as there are more orbits available than the current allotment and assignment of GEO belt slots.

Additionally, our analysis revealed that the the space vehicle cost is the largest contributor to the LCOEs for both MEO and GEO, outweighing both the launch and ground segment costs. Consequently, reducing the space vehicle mass, which generally reduces both manufacturing and launch costs, and to a lesser extent, increasing the power transmission efficiency, have the greatest potential to decrease the cost of a space solar power system. This is contrary to the widespread assumption in the literature, namely that these systems will achieve economic viability with an order of magnitude reduction in launch costs. In other words, development of a low-cost space vehicle is paramount for making space solar power economically viable; launch and ground segment costs are significant but secondary.

Lastly, it is noted that the parametric model we use has not been calibrated to our system. It has its origins in NASA cost data [44] for more traditionally designed satellite systems, and thus provides a relative measure of cost for our system. That is to say, without calibration using actual cost data pertaining to an SSPP-like satellite, the parametric cost, and hence the LCOE that results from using that cost, is a relative figure of merit for comparing system instantiations. Since the same cost models are used regardless of the system instantiation, the resulting LCOE is a valid figure of merit to use for comparing different systems. However, the predicted LCOE may not be a realistic indication of what a consumer might actually pay for energy from a space solar power system. Thus, while we use LCOE to compare instantiations, we do not assert that these LCOEs would be the ones realized by actual systems.

\section{ACKNOWLEDGMENT}

M. A. Marshall was supported by a NASA Space Technology Research Fellowship. Financial support from the Caltech 
Space Solar Power Project is also gratefully acknowledged. We thank Profs. Harry Atwater and Ali Hajimiri, Drs. Dan Scharf, Mike Kelzenberg, Emily Warmann, and Terry Gdoutos, and Mr. Austin Fikes for insightful discussions on space solar power. We likewise thank two anonymous reviewers for their comments and suggestions that improved the paper.

\section{REFERENCES}

[1] I. Asimov, "Reason," in I, Robot, 1941, pp. 59-77.

[2] J. C. Mankins, The Case for Space Solar Power. Houston, Texas: Virginia Edition Publishing, 2014.

[3] National Space Society, "Space Solar Power - Library," National Space Society. [Online]. Available: https://space.nss.org/ space-solar-power-library/ [Accessed Apr. 11, 2019].

[4] P. E. Glaser, "Power from the Sun: Its Future," Science, vol. 162, no. 3856, pp. 857-861, 1968.

[5] J. C. Mankins, "A fresh look at space solar power: New architectures, concepts and technologies," Acta Astronautica, vol. 41, no. 4, pp. 347359, 1997.

[6] C. Carrington, J. Fikes, M. Gerry, D. Perkinson, H. Feingold, and J. Olds, "The Abacus/Reflector and Integrated Symmetrical Concentrator: Concepts for Space Solar Power Collection and Transmission," in 35th Intersociety Energy Conversion Engineering Conference and Exhibit, Las Vegas, Nevada, 2000, AIAA-2000-3067.

[7] J. C. Mankins, "A Technical Overview of the "SunTower" Solar Power Satellite Concept," Acta Astronautica, vol. 50, no. 6, pp. 369-377, 2002.

[8] S. Sasaki, K. Tanaka, K. Higuchi, N. Okuizumi, S. Kawasaki, N. Shinohara, K. Senda, and K. Ishimura, "A new concept of solar power satellite: Tethered-SPS,” Acta Astronautica, vol. 60, no. 3, pp. 153-165, 2007.

[9] M. Arya, N. Lee, and S. Pellegrino, "Ultralight Structures for Space Solar Power Satellites," in 3rd AIAA Spacecraft Structures Conference, San Diego, California, 2016, AIAA-2016-1950.

[10] X. Li, B. Duan, L. Song, Y. Yang, Y. Zhang, and D. Wang, "A new concept of space solar power satellite," Acta Astronautica, vol. 136, pp. 182-189, 2017.

[11] I. Cash, "Cassiopeia - a new paradigm for space solar power," Acta Astronautica, vol. 159, pp. 170-178, 2019.

[12] R. Jehn, V. Agapov, and C. Hernández, "The situation in the geostationary ring," Advances in Space Research, vol. 35, no. 7, pp. 1318-1327, 2005.

[13] W. J. Larson and J. R. Wertz, Space Mission Analysis and Design, 3rd ed. El Segundo, California and Dordrecht, The Netherlands: Microcosm Press and Kluwer Academic Publishers, 1999, ch. 7, 8, pp. 159-240.

[14] R. G. Madonna, "Use of an Iterative Research and Development System Engineering Approach for the Caltech Space Solar Power Project," in 6th IEEE International Conference on Wireless for Space and Extreme Environments (WiSEE), Huntsville, Alabama, 2018, pp. 200-205.

[15] M. A. Marshall, A. Goel, and S. Pellegrino, "Power-Optimal Guidance for Planar Space Solar Power Satellites," Journal of Guidance, Control, and Dynamics, vol. 43, no. 3, pp. 518-535, 2020.

[16] E. E. Gdoutos, C. Leclerc, F. Royer, M. D. Kelzenberg, E. C. Warmann, P. Espinet-Gonzalez, N. Vaidya, F. Bohn, B. Abiri, M. R. Hashemi, M. Gal-Katziri, A. Fikes, H. Atwater, A. Hajimiri, and S. Pellegrino, "A Lightweight Tile Structure Integrating Photovoltaic Conversion and RF Power Transfer for Space Solar Power Applications," in AIAA SciTech Forum, Kissimmee, Florida, 2018, AIAA-2018-2022.

[17] M. R. M. Hashemi, A. C. Fikes, M. Gal-Katziri, B. Abiri, F. Bohn, A. Safaripour, M. D. Kelzenberg, E. L. Warmann, P. Espinet, N. Vaidya, E. E. Gdoutos, C. Leclerc, F. Royer, S. Pellegrino, H. A. Atwater, and A. Hajimiri, "A flexible phased array system with low areal mass density," Nature Electronics, vol. 2, pp. 195-205, 2019.

[18] E. C. Warmann, P. Espinet-Gonzalez, N. Vaidya, S. Loke, A. Naqavi, T. Vinogradova, M. Kelzenberg, C. Leclerc, E. Gdoutos, S. Pellegrino, and H. A. Atwater, "An ultralight concentrator photovoltaic system for space solar power harvesting," Acta Astronautica, vol. 170, pp. 443-451, 2020.

[19] H. Matsumoto, "Research on Solar Power Satellites and Microwave Power Transmission in Japan," IEEE Microwave Magazine, vol. 3, no. 4, pp. 36-45, 2002.

[20] P. Jaffe and J. McSpadden, "Energy conversion and transmission modules for space solar power," Proceedings of the IEEE, vol. 101, no. 6, pp. 1424-1437, 2013.
[21] W. C. Brown, "The history of power transmission by radio waves," IEEE Transactions on Microwave Theory and Techniques, vol. 32, no. 9, pp. $1230-1242,1984$

[22] W. C. Brown and E. E. Eves, "Beamed microwave power transmission and its application to space," IEEE Transactions on Microwave Theory and Techniques, vol. 40, no. 6, pp. 1239-1250, 1992.

[23] S. Sasaki, K. Tanaka, and K.-I. Maki, "Microwave power transmission technologies for solar power satellites," Proceedings of the IEEE, vol. 101 , no. 6, pp. 1438-1447, 2013

[24] Z. J. Silva, C. R. Valenta, and G. D. Durgin, "Optically Transparent Antennas: A Survey of Transparent Microwave Conductor Performance and Applications," IEEE Antennas and Propagation Magazine, vol. 63, no. 1, pp. 27-39, 2021.

[25] H. Qiu, H. Liu, X. Jia, Z.-Y. Jiang, Y.-H. Liu, J. Xu, T. Lu, M. Shao, T.L. Ren, and K. J. Chen, "Compact, Flexible, and Transparent Antennas Based on Embedded Metallic Mesh for Wearable Devices in 5G Wireless Network," IEEE Transactions on Antennas and Propagation, vol. 69, no. 4, pp. 1864-1873, 2021.

[26] R. N. Simons and R. Q. Lee, "Feasibility study of optically transparent microstrip patch antenna," in IEEE Antennas and Propagation Society International Symposium, Montreal, Quebec, Canada, 1997, pp. 21002103.

[27] T. W. Turpin and R. Baktur, "Meshed Patch Antennas Integrated on Solar Cells," IEEE Antennas and Wireless Propagation Letters, vol. 8, pp. 693-696, 2009.

[28] C. G. M. Ryan and G. V. Eleftheriades, "Single- and Dual-Band Transparent Circularly Polarized Patch Antennas With Metamaterial Loading," IEEE Antennas and Wireless Propagation Letters, vol. 14, pp. 470-473, 2014.

[29] A. Pedivellano, E. Gdoutos, and S. Pellegrino, "Sequentially controlled dynamic deployment of ultra-thin shell structures," in AIAA Scitech Forum, Orlando, Florida, 2020, AIAA 2020-0690.

[30] E. Gdoutos, C. Leclerc, F. Royer, D. A. Türk, and S. Pellegrino, "Ultralight Spacecraft Structure Prototype," in AIAA Scitech Forum, San Diego, California, 2019, AIAA-2019-1749.

[31] E. Gdoutos, A. Truang, A. Pedivellano, F. Royer, and S. Pellegrino, "Ultralight Deployable Space Structure Prototype," in AIAA Scitech Forum, Orlando, Florida, 2020, AIAA-2020-0692.

[32] A. Goel, N. Lee, and S. Pellegrino, "Trajectory design of formation flying constellation for space-based solar power," in IEEE Aerospace Conference, Big Sky, Montana, 2017.

[33] A. Goel, S.-J. Chung, and S. Pellegrino, "Trajectory Design of a Spacecraft Formation for Space-Based Solar Power Using Sequential Convex Programming," in Proceedings of the 9th International Workshop on Satellites Constellations and Formation Flying (IWSCFF), Boulder, Colorado, 2017.

[34] P. R. Escobal, Methods of Orbit Determination, 2nd ed. Malabar, Florida: Krieger Publishing Company, Inc., 1976, ch. 4.

[35] C. R. Ortizlongo and S. L. Rickman, "Method for the calculation of spacecraft umbra and penumbra shadow terminator points," NASA Lyndon B. Johnson Space Center, Houston, Texas, Tech. Rep. NASATP-3547, 1995.

[36] C. A. Balanis, Antenna Theory: Analysis and Design, 3rd ed. Hoboken, New Jersey: John Wiley \& Sons, Inc., 2005, ch. 6, pp. 283-333.

[37] M. A. Marshall, A. Goel, and S. Pellegrino, "Attitude maneuver design for planar space solar power satellites," in 29th AAS/AIAA Space Flight Mechanics Meeting, Ka'anapali, Maui, Hawaii, 2019, AAS 19-287.

[38] K. R. Raney, A. P. Luscombe, E. J. Langham, and S. Ahmed, "RADARSAT (SAR imaging)," Proceedings of the IEEE, vol. 79, no. 6, pp. 839-849, 1991.

[39] G. W. Davidson and I. Cumming, "Signal properties of spaceborne squint-mode SAR," IEEE Transactions on Geoscience and Remote Sensing, vol. 35, no. 3, pp. 611-617, 1997.

[40] P. Jaffe, "The Opportunity of Space Solar," in Future In-Space Operations Telecon, Dec. 14, 2016. [Online]. Available: http://fiso.spiritastro. net/archivelist.htm [Accessed Mar. 29, 2019].

[41] SpaceX, Falcon User's Guide, January 2019. [Online]. Available: https://www.spacex.com/sites/spacex/files/falcon_users_guide_0219.pdf

[42] A. B. Badiru, "Computational survey of univariate and multivariate learning curve models," IEEE Transactions on Engineering Management, vol. 39, no. 2, pp. 176-188, 1992.

[43] E. Hecht, Optics, 3rd ed. Boston, Massachusetts: Addison-Wesley Longman, 1997, ch. 10, pp. 350-353.

[44] D. C. Arney and A. W. Wilhite, "Rapid Cost Estimation for Space Exploration Systems," in AIAA SPACE Conference \& Exposition, Pasadena, California, 2012, AIAA-2012-5183. 
[45] Analytical Graphics, Inc., "Systems Tool Kit (STK), version 11.5.0," Exton, Pennsylvania.

[46] E. Donchev, J. S. Pang, P. M. Gammon, A. Centeno, F. Xie, P. K. Petrov, J. D. Breeze, M. P. Ryan, D. J. Riley, and N. M. Alford, "The rectenna device: From theory to practice (a review)," MRS Energy \& Sustainability, vol. 1, p. E1, 2014.

[47] Wikipedia.org, "Tesla Powerwall," Wikipedia.org, Feb. 13, 2019. [Online]. Available: https://en.wikipedia.org/wiki/Tesla_Powerwall [Accessed Mar. 2019].

[48] U.S. Energy Information Administration, "Frequently asked questions - how much electricity does an American home use?" U.S. Energy Information Administration, Oct. 26, 2018. [Online]. Available: https: //www.eia.gov/tools/faqs/faq.php?id=97\&t=3 [Accessed 15 Apr. 2019].

[49] Wikipedia.org, "Comparison of orbital launch systems," Wikipedia.org, Mar. 17, 2019. [Online]. Available: https://en.wikipedia.org/wiki/ Comparison_of_orbital_launch systems. [Accessed Mar. 2018].

[50] M. A. Green, A. Ho-Baillie, and H. J. Snaith, "The emergence of perovskite solar cells," Nature Photonics, vol. 8, pp. 506-514, 2014.

[51] J.-S. Huang, M. D. Kelzenberg, P. Espinet-González, C. Mann, D. Walker, A. Naqavi, N. Vaidya, E. Warmann, and H. A. Atwater, "Effects of Electron and Proton Radiation on Perovskite Solar Cells for Space Solar Power Application," in 2017 IEEE 44th Photovoltaic Specialist Conference (PVSC), Washington, DC, 2017, pp. 1248-1252.

[52] Y. Miyazawa, M. Ikegami, H.-W. Chen, T. Ohshima, M. Imaizumi, K. Hirose, and T. Miyasaka, "Tolerance of Perovskite Solar Cell to HighEnergy Particle Irradiations in Space Environment," iScience, vol. 2, pp. 148-155, 2018.

[53] I. Cardinaletti, T. Vangerven, S. Nagels, R. Cornelissen, D. Schreurs, J. Hruby, J. Vodnik, D. Devisscher, J. Kesters, J. D'Haen, A. Franquet, V. Spampinato, T. Conard, W. Maes, W. Deferme, and J. V. Manca, "Organic and perovskite solar cells for space applications," Solar Energy Materials and Solar Cells, vol. 182, pp. 121-127, 2018.

[54] B. E. Anspaugh, "Proton and electron damage coefficients for $\mathrm{GaAs} / \mathrm{Ge}$ solar cells," in The Conference Record of the Twenty-Second IEEE Photovoltaic Specialists Conference - 1991, vol. 2, Las Vegas, Nevada, 1991, pp. 1593-1598.

[55] Land Watch. [Online]. Available: https://www.landwatch.com/Hawaii land_for_sale/Maui_County/Land [Accessed Mar. 26, 2019].

[56] Land Watch. [Online]. Available: https://www.landwatch.com/Arizona land_for_sale/Land [Accessed Mar. 26, 2019].
Michael A. Marshall received the B.S. in Aerospace Engineering from the Georgia Institute of Technology, Atlanta, GA in 2016 and the M.S. in Space Engineering from the California Institute of Technology, Pasadena, CA in 2017. He is currently a NASA Space Technology Research Fellow working towards his Ph.D. in Space Engineering at Caltech. His research focuses on the dynamics and control of ultralight, flexible spacecraft.

Richard G. Madonna (M'86-SM'04) received the B.S. in physics from Stevens Institute of Technology, Hoboken, NJ, the M.S. in physics from Adelphi University, Garden City, NY, the Ph.D. in physics from Stevens Institute of Technology, and the M.B.A. from Long Island University, Greenlawn, NY. From 1982-1986, he served on active duty as Captain, USAF at the USAF Technical Applications Center where he conducted R\&D in support of national security missions. Of note, he oversaw the development of a Space Shuttle mid-deck payload experiment which flew 10 times from 1983-1986. He joined Grumman Aerospace Corporate Research Center in 1986, where he developed high power RF sources, impulse radar sources, and eventually initiated and lead the Remote Sensing program. In 1998, Rich transferred to Northrop Grumman's Electronic Systems Sector (now called Mission Systems) in Baltimore, Maryland where he served as System Architect on several spacebased EO and IR sensing programs supporting defense and national security efforts. In 2015, Rich was named as Northrop Grumman's System Architect for the NGC/Caltech Space Solar Power Initiative. He retired from NGC in 2017 and joined the Caltech SSPP team as Project Manager in 2018.

Dr. Madonna is the author of more than 40 papers and the author of one book, Orbital Mechanics. He has held adjunct faculty positions at Florida Institute of Technology, Rollins College, Hofstra University, Johns Hopkins Whiting School of Engineering, and is currently an adjunct professor at University of Maryland Baltimore County. He is a member of IEEE, SPIE, OSA, and Sigma Xi.
Sergio Pellegrino received the Laurea degree in civil engineering from the University of Naples, Naples, Italy in 1982, and the Ph.D. degree in structural mechanics from the University of Cambridge, Cambridge, U.K., in 1986. He served on the faculty of the University of Cambridge from 1985 to 2007. $\mathrm{He}$ is currently the Joyce and Kent Kresa Professor of Aerospace and Civil Engineering at the California Institute of Technology, Pasadena, CA, USA, a Jet Propulsion Laboratory Senior Research Scientist, and a Co-Director of the Caltech Space Solar Power Project. His current research interests include the mechanics of lightweight structures with a focus on packaging, deployment, shape control, and stability. With his students and collaborators, he is currently working on novel concepts for future space telescopes, spacecraft antennas, and space-based solar power systems. Dr. Pellegrino is a Fellow of the Royal Academy of Engineering, a Fellow of AIAA, and a Chartered Structural Engineer. He is President of the International Association for Shell and Spatial Structures (IASS) and was the founding chair of the AIAA Spacecraft Structures Technical Committee. Dr. Pellegrino has authored over 300 technical publications and has recently co-authored the book Forms and Concepts for Lightweight Structures. 